\title{
Kapitel 1 - Theoretische Befunde zum Begriff der Abwehr
}

Im vorliegenden Kapitel werden Befunde verschiedener theoretischer Disziplinen mit dem Ziel vorgestellt, eine soziologische Analytik der Abwehr von Thematisierungen und Problematisierungen von Antisemitismus zu entwickeln. Im Kern geht es hierbei darum, Abwehr als Kommunikationsphänomen soziologisch beschreib- und verstehbar zu machen. Ich werde argumentieren, dass jedes (Kommunikations-)Verhalten, das darauf zielt, Diskurse über die Problematik antisemitischer Ereignisse und Handlungen gar nicht erst aufkommen zu lassen oder einen entsprechenden Diskurs so zu beschneiden, dass es zu einer weitgehenden Entschärfung oder Neutralisierung von antisemitischen Konflikten kommt, als Abwehrphänomene bezeichnet werden kann. Darüber hinaus werde ich herausstellen, dass die gesellschaftliche Normalisierung von Abwehrhandlungen auch zu einer Prägung kulturell verankerter Überzeugungen führen kann, die dann den Verlauf von antisemitischen Konflikten signifikant beeinflussen.

Um dies zu plausibilisieren, werden nicht allein Befunde der Antisemitismusforschung referiert. Ergänzend werden eine Reihe theoretischer und empirischer Forschungsarbeiten rezipiert, die Abwehrhandlungen in anderen thematischen Kontexten zum Gegenstand haben. Hierbei zeigt sich, dass bestimmte Aspekte des Phänomens, die in einem Forschungsansatz eher randständig thematisiert werden, in anderen von größerer Bedeutung sind. $\mathrm{Zu}$ gleich lässt sich in der Zusammenschau zeigen, dass die verschiedenen Ansätze in vielerlei Hinsicht aufeinander konvergieren. Auf die verschiedenen Formen der Abwehr, die in Kapitel 2 portraitiert werden, gehe ich in diesen Darstellungen nur am Rande ein, um die Befunde der jeweiligen Theorieansätze illustrativ zu verdeutlichen.

Da der Begriff der Abwehr vor allem durch die psychoanalytische Forschung geprägt worden ist und sich für weitere Bestimmungsversuche als 
modellbildend erweist, erscheint es sinnvoll, diese Beiträge als Ausgangspunkt für weitere theoretische Bestimmungsversuche zu nutzen. In der psychoanalytischen Forschung wird Abwehr vor allem als Form der Bewältigung von psychologischen Konflikten gefasst. Damit einher geht die Überlegung, dass Abwehrprozesse dazu dienen, psychische Systeme in Krisenlagen zu stabilisieren (Kapitel 1.1.). Demgegenüber lässt sich in Anschluss an das in der Antisemitismusforschung einschlägige Konzept der Kommunikationslatenz zeigen, dass sich Abwehr auch sozialwissenschaftlich konzeptualisieren lässt. Diesbezüglich argumentiere ich, dass das Konzept der >Latenz des Antisemitismus`, welches üblicherweise herangezogen wird, um eine spezifische Form der Abwesenheit des Antisemitismus in der öffentlichen Kommunikation zu erklären, auch produktiv auf die Untersuchung von Abwehrstrategien angewendet werden kann. Unter Latenzkommunikation werden in diesem Sinne funktionale Tätigkeiten verstanden, die dazu dienen, ein politisches System durch das Abdrängen von Antisemitismuskritik nach innen und außen zu stabilisieren (Kapitel 1.2). Demgegenüber wird in Anschluss an Forschungsergebnisse der Kriminologen Gresham M. Sykes und David Matza (Kapitel 1.3) sowie das von Friedrich Pollock für das Frankfurter Institut für Sozialforschung durchgeführte "Gruppenexperiment« (Kapitel 1.4) deutlich, dass sich Abwehr auch als eine mit Sinn versehene, soziale Handlung verstehen lässt, die sowohl psychische Funktionen (Entlastung des Gewissens) als auch soziale Funktionen (Sicherstellung sozialer Anerkennung) zu erfüllen vermag. In beiden Kapiteln wird herausgearbeitet, dass durch Abwehrhandlungen sozial geltende Normen und Werte auf häufig flüchtige und unauffällige Weise unterlaufen werden und Abwehrhandlungen als verdeckt instrumentelle Handlungen betrachtet werden müssen. In den Kapiteln 1.6 und 1.7 werden die bis hierhin entwickelten Überlegungen dann mit sprechakttheoretischen Ansätzen in Verbindung gebracht. Dies umfasst die Zusammenfassung von Ergebnissen aus dem Feld der diskursiven Psychologie, im Kontext dessen verschiedene Autor*innen argumentative Formen der Abwehr im Kontext alläglicher Auseinandersetzungen über politische Reizthemen - insbesondere Rassismus - analysiert haben. Hier werden Abwehrhandlungen als Sprechhandlungen gefasst, die rassistisch sprechenden Akteuren dazu dienen, in Diskurszusammenhängen ihre soziale Identität gegen Rassismuskritik zu immunisieren. Weiterhin wird der sprechakttheoretisch-fundierte Ansatz zu Kommunikationspathologien von Jürgen Habermas (1984) rezipiert, in dem das psychologische Konzept der Abwehr kommunikationstheoretisch weiterentwickelt wird. Hier werden unter Abwehrhandlungen verschiedene 
Formen von oftmals subtilen Kommunikationsabbrüchen gefasst, mit denen ein Konfliktpotenzial in zwischenmenschlichen Beziehungen stillgestellt wird und die in diesem Sinne soziale Funktionen erfüllen. Das Kapitel zu den theoretischen Bestimmungsversuchen von Abwehrhandlungen wird dann in Kapitel 1.7 durch die Diskussion von Birgit Rommelspachers psychoanalytischer Studie "Schuldlos Schuldig« (1995) abgeschlossen und gezeigt, wie sich die allgemein gehaltenen Überlegungen Jürgen Habermas' auf das Feld der Antisemitismusforschung beziehen lassen. Deutlich wird hierbei insbesondere, dass Abwehrhandlungen nicht nur für antisemitisch handelnde Personen, sondern auch für Menschen, die diesen persönlich nahestehen, von Bedeutung sein können.

\subsection{Abwehr als Konzept der Metapsychologie}

Wie bereits in der Einleitung festgestellt wurde, stammt der Terminus $>\mathrm{Ab}$ wehr<, wie er in der Antisemitismusforschung häufig genutzt wird, aus der psychologischen Theorie Sigmund Freuds (im Folgenden als Metapsychologie bezeichnet ${ }^{1}$ ). Der Psychoanalytiker hatte ihn in seinen frühen Schriften eingeführt, um den Umgang des Ichs mit Triebansprüchen zu beschreiben, die nicht bewusst werden sollen und deshalb von der möglichen Versprachlichung ausgeschlossen werden (vgl. Ritter 2008: 119). Später ersetzte Freud den Begriff dann zunächst durch den der »Verdrängung ", um diese zuletzt als eine bestimmte Abwehrmethode neben etlichen anderen $\mathrm{zu}$ bezeichnen (Freud 1971: 302). Abwehrmechanismen werden hierbei als Ich-Tätigkeiten verstanden, die zu großen Teilen unbewusst verlaufen und als »Vorgänge mit gleicher Tendenz - Schutz des Ichs gegen Triebansprüche« definiert werden (ebd.: 301).

Der Abwehrbegriff wurde insbesondere von Anna Freud in »Das Ich und die Abwehrmechanismen " (Freud 2003) in systematisierender Absicht aufgenommen und weiterentwickelt. Abwehrmechanismen werden von ihr nicht choanalyses, die eine Untersuchungsmethode oder therapeutische Methode bezeichnet - die begrifflichen Modelle, theoretischen Annahmen und allgemeinen Hypothesen beschrieben, die in der Abstraktion von konkreten Beobachtungen gewonnen werden (vgl. Laplanche/Pontalis 1973: 307). 
allein als Umgangsweisen mit Trieben, sondern als Oberbegriff für alle Mittel verstanden, "mit denen das Ich sich gegen Unlust und Angst verteidigt« (ebd.: 9). Für das Ich irritierend könnten demnach z.B. auch Gefühle der Entsagung, Minderwertigkeit, Trauer oder Aggression sein. In systematischer Hinsicht führte die Arbeit Anna Freuds dazu, deutlicher zwischen den vielen unterschiedlichen psychischen Konstellationen und Motiven, die Abwehr zugrunde liegen, zu unterscheiden. So erscheint z.B. bei der »Triebabwehr aus Über-Ich-Angst« ein Trieb dem Ich als gefährlich, da seine Erfüllung einen Konflikt zwischen dem Ich und dem Über-Ich als Gewissensinstanz provozieren und Schuldgefühle auslösen würde. Gefürchtet wird also nicht der Trieb selbst, sondern die verurteilende Instanz, die einen »Druck « (ebd.: 61) auf das Ich ausübt. Demgegenüber wird unter der »Triebabwehr aus Realangst« ein abwehrendes Verhalten beschrieben, das aus der Androhung von Strafen und Einschränkungen (insbesondere durch die Eltern) resultiere. Hier erfolgte die Abwehr »unter dem Druck der Angst vor der Außenwelt« (ebd.: 63.).

Dem metapsychologischen Konzept zufolge verläuft der Abwehrprozess selbst »stumm und unsichtbar«. Der Vollzug der Abwehr durch die Abwehrmechanismen ließe sich demnach zwar "nachträglich rekonstruieren«, nie aber »wirklich verfolgen« (ebd.: 17). Sicht- bzw. im therapeutischen Prozess kommunizierbar seien hingegen "Ersatzbildungen«, die durch die meisten Abwehrmechanismen hervorgebracht werden (vgl. Zepf 2012: 54f. ${ }^{2}$ ). Diese werden als »verschlüsselte Darstellungen« (ebd.: 55) des Abgewehrten betrachtet, die das »unbewusst Gewordene wieder in entstellter Weise ins Bewusstsein einfügen« (ebd.: 55). Ersatzbildungen zeichnen sich demnach dadurch aus, dass sie ich-synton sind, d.h. sich anders als die abgewehrten Vorstellungen, Wünsche und Ängste in das Ich integrieren lassen. Solche Ersatzbildungen werden dementsprechend auch als Formen der »Selbsttäuschung« (vgl. ebd.: 53) verstanden. Der Begriff der Rationalisierung bezieht sich sodann auf den Gebrauch von Ersatzbildungen. Indem für diese rational nachvollziehbare Gründe gesucht werden, um sie gegen ihre Infragestellung abzusichern, verschleiern Rationalisierungen den dem Abwehrmechanismus zugrundeliegenden Konflikt auf sekundäre Weise (vgl. Laplanche/Pontalis

Dies gilt Freud zufolge mit Ausnahme des Mechanismus der Verdrängung, die »der zurückgewiesenen Vorstellung [...] [d]ie Übersetzung in Worte verweigert, womit die nicht in Worte gefasste Vorstellung [...] dann im Ubw als verdrängt zurück bleibt« (Freud 1915: 300, zitiert nach ebd.: 54). 
1973: 418). Ein solcher Vorstellungsgehalt kann dann auch intellektualisiert, d.h. mit hoch abstrakten Schein-Begründungen versehen werden.

Was eine solche Konzeptualisierung der Abwehr für die Antisemitismusforschung konkret bedeuten kann, soll hier an mehreren Beispielen illustriert werden. So ist der Begriff z.B. für die Analyse des Antisemitismus selbst fruchtbar gemacht worden (vgl. Salzborn 2010, Beland 2004). In Anschluss an Ernst Simmel, Béla Grunberger, Otto Fenichel und andere wird argumentiert, dass Antisemitismus psychologisch als Resultat des Abwehrmechanismus der Projektion verstanden werden sollte: "Sozial induzierte, unerträglich gewordene intrapsychische Spannungen« (Pohl 2010: 43) und damit verbundene Aggressionen gegen sich und die Außenwelt werden demnach bewältigt, indem sie auf Vorstellungen von Jüd*innen oder jüdischen Institutionen übertragen und anschließend in verzerrter Weise als äußere Gefahr (wieder)erkannt werden. Aggressionen, die eigentlich anderen Objekten gelten, werden also in Hass gegen Jüd*innen transformiert (vgl. Pollock 1955: 232). Damit korrespondierende Vorstellungen von Jüd*innen als Gefahr sind nun für das Ich annehmbar und können z.B. im Zuge einer Entfaltung als Verschwörungstheorien immer weiter intellektualisiert werden. Dadurch werden nicht nur Wahnideen plausibilisiert, sondern auch die Wahrscheinlichkeit möglichen inneren Widerspruchs hiergegen herabgesetzt und somit das Ich gegen Infragestellungen des Gewissens abgeschirmt. Die Abwehr der (selbst)kritischen Reflexion des eigenen Antisemitismus als gewalttätige Zurichtung der Realität ist demnach aus metapsychologischer Perspektive bereits in den Prozess der psychischen Bildung antisemitischer Ideologeme eingebaut.

Weiterhin ist der Begriff der Abwehr auch für die Analyse des Umgangs mit vergangenem Antisemitismus fruchtbar gemacht worden. Ein Klassiker in diesem Forschungsfeld ist die von Margarete und Alexander Mitscherlich durchgeführte Studie »Die Unfähigkeit zu trauern« (1977). Hier wird die in der Nachkriegszeit verbreitete Weigerung, sich reflexiv mit der nationalsozialistischen Vergangenheit zu beschäftigen, als Resultat der Abwehr von Gefühlen der Angst, der Schuld und Scham, sowie einer drohenden Identitätskrise verstanden. Gefühle der »Vergeltungsangst « resultierten demnach aus dem Wissen um den von Deutschland angezettelten Krieg und der nationalsozialistischen Verbrechen, Gefühle der Schuld aus dem Wissen um eigene Gewalttaten oder die Duldung der Taten anderer (vgl. ebd.: 2off., vgl. auch Giordano 1990: 9ff., Schwan 1990: 101ff.) und Gefühle der Scham aus dem Wissen um den tiefen Fall des sozialen Ansehens der Deutschen. Demgegenüber drohte den Mitscherlichs zufolge auch eine massive Herabsetzung 
des »Ich-Gefühls«, da die idealisierte Figur Adolf Hitlers im Nationalsozialismus als Ersatz für das eigene Ich-Ideal (dem als erstrebenswert betrachteten Selbstbild als Teil des Über-Ichs) gedient habe. Anstatt sich diesen Problemen allerdings selbstkritisch zu stellen, hätten die Abwehrmechanismen der »Derealisierung der Vergangenheit«, des »Ungeschehenmachens « im Versuch des Wiederaufbaus und der »Identifikation« mit den alliierten Siegern dazu gedient, die bedrohlichen psychischen Folgen der Deutschen Niederlage zu bewältigen (Mitscherlich/Mitscherlich 1967: 56ff.). ${ }^{3}$

Auch für die Analyse des sogenannten sekundären oder Schuldabwehrantisemitismus spielt der Begriff der Abwehr eine große Rolle (vgl. z.B. Stender 2011, Bergmann 2007, Höttemann 2020). Hinter den für dessen Beschreibung oftmals benutzten Wendung, dass es sich hierbei um einen Antisemitismus "nicht trotz, sondern wegen Auschwitz« (Broder 1986: 37) handle, verbergen sich allerdings unterschiedliche (psychologische und soziologische) Konzeptualisierungsversuche, die in Kapitel 2.9 ausführlich diskutiert werden.

Abschließend sei darauf hingewiesen, dass die in der Metapsychologie konstitutive Idee, dass Abwehr als Form der Bewältigung von innerpsychischen Konflikten zu verstehen sei, auch in der Medienwirkungsforschung adaptiert worden ist. So wird im Kontext der Diskussion über das Theorem des Hostile Media Phenomenon (vgl. Lord et al. 1979: 2099) davon ausgegangen, dass ein Publikum mit starken Voreinstellungen zu bestimmten Themen auf mediale Erzeugnisse dann mit Feindseligkeit reagiert, wenn diese sie nicht in ihren Einstellungen bestärken und deshalb als Bedrohung wahrgenommen werden. Einer quantitativen Studie von Bergmann/Erb (1991b) zufolge, stilisieren dementsprechend insbesondere Menschen mit antisemitischen Einstellungen die Medien zu manipulativen Akteuren und bringen dies mit einer vermeintlichen >linken oder >jüdischen Macht< oder einer >jüdischen Verschwörung in Verbindung. Das Problem einer »kognitiven Dissonanz«, die aus der Akzeptanz einer anti-antisemitischen öffentlichen Meinung entstehen müsste, wird hierbei neutralisiert, indem ein psychologisch annehmbares »Täuschungskonzept « entwickelt wird, »das den Massenmedien und den Politikern unterstellt, die >Wahrheit und die >wirkliche Meinung

Vgl. hierzu auch die Ausführungen Markus Brunners (2011), der die von den Mitscherlichs aufgestellten Hypothesen sowie der Kritik hieran einer kritischen Würdigung unterzieht und unter Berücksichtigung aktueller Diskussionen die von den Psychoanalytikerinnen beschriebenen Prozesse neu beleuchtet. 
einer äußeren Einflussnahme (etwa Seitens >der Juden und der Westmächte)« (vgl. ebd.: 509) zu unterdrücken.

\section{Zwischenfazit}

In den vorhergehenden Abschnitten wurde die Verwendung des metapsychologischen Konzepts der Abwehr in der Antisemitismusforschung ausgeführt. Da sich im Fortgang der folgenden Kapitel der somit umrissene Begriff in vielerlei Hinsichten als modellbildend erweist, sollen nun abschließend vor allem die allgemeinen Spezifika des Konzepts zusammengefasst werden. Diesbezüglich lässt sich festhalten, dass der Begriff der Abwehr für die Metapsychologie konstitutiv ist. Abwehrmechanismen werden als funktionale Tätigkeiten des psychischen Systems betrachtet, mit dem Konflikte und Bedrohungen, die aus Widersprüchen im System und Spannungen, die durch Druck von außen entstehen, innerhalb des Systems selbst bewältigt werden. Abwehrmechanismen dienen hierbei dazu, Konflikte »unbewusst zu machen oder unbewußt zu halten« (Mentzos 2010: 60), d.h. sie beziehen sich sowohl auf manifeste wie auch latente psychische Konflikte. Die damit verbundene Bewältigung ist allerdings unvollständig und stets vorläufig, weil sie nur einen Schein der Gefahrenbewältigung oder Konfliktlösung und nicht die Lösung selbst hervorbringt. Aus diesem Grunde können durch Abwehrmechanismen produzierte Vorstellungsinhalte auch als Form der Selbsttäuschung verstanden werden, deren psychologischer Gewinn darin liegt, dass sie sich auf unproblematische Weise in das eigene Selbstverständnis integrieren lassen. Die Analyse der Abwehr zielt hierbei letztlich auf die »tiefenhermeneutische Erfassung menschlicher Weil-Motive« (Eberle 1984: 266), d.h. sie unterstellt Motive, Gründe und einen (unbewussten) Sinn, der abwehrenden Handlungen zugrunde läge. Dementsprechend besteht die Arbeit der psychoanalytischen Praxis in der »Rückgewinnung« des verdrängten Motivs: »Analytische Kritik ist Kritik an der Abwehr; an der verdrängenden Instanz« (Küchenhoff 2009: 306).

Nun lässt sich aus soziologischer Perspektive fragen, ob sich dieses Konzept auch sozialwissenschaftlich konzeptualisieren lässt. Wenn dem so wäre, müsste gezeigt werden können, dass die Abwehr der Thematisierung von vergangenem oder gegenwärtigem Antisemitismus nicht nur interne psychologische, sondern auch externe soziale Funktionen erfüllt. Abwehr würde dann nicht als psychische, sondern als soziale Tätigkeit zu beschreiben sein. Dass dies durchaus möglich ist, soll nun in einem ersten Schritt in Auseinander- 
setzung mit einem Ansatz aus der Antisemitismusforschung gezeigt werden, der sich zu diesem Zweck systemtheoretische Überlegungen zu eigen macht.

\subsection{Abwehr als Latenzkommunikation}

Mit der Darstellung und Interpretation des für die Antisemitismusforschung einschlägigen Theorems der Kommunikationslatenz der Soziologen Werner Bergmann und Rainer Erb (vgl. Bergmann/Erb 1986) kann eine genuin soziologische Konzeptualisierung der Abwehr von Thematisierungen von Antisemitismus vorgestellt werden. Diese ähnelt zwar der metapsychologischen Theorie, kommt allerdings in weiten Teilen ohne subjekttheoretische Überlegungen aus. Das Theorem der Kommunikationslatenz mit einer Theorie der Abwehr in Verbindung zu bringen, mag dabei auf den ersten Blick überraschend erscheinen, insofern es von Bergmann insbesondere im Zusammenhang mit einer Theorie kollektiven Lernens formuliert worden ist (vgl. Bergmann 1997: 495ff., 2002, vgl. auch Miller 1990). Deren Fokus wiederum liegt nicht auf der Abwehr der Thematisierung von Antisemitismus, sondern auf der stetig voranschreitenden Durchsetzung einer anti-antisemitischen Kommunikationsnorm nach Auschwitz, die nicht nur zu einem weitgehenden Verschwinden von Antisemitismus in der Öffentlichkeit geführt, sondern auch die Abnahme antisemitischer Einstellungen in der Bevölkerung bedingt habe. Diese Entwicklung wird als Resultat einer langen Kette von antisemitischen Konflikten dargestellt, in denen antisemitische Ereignisse stets aufs Neue öffentlich problematisiert worden sind (vgl. Bergmann 1997: 15). Ich werde diesbezüglich argumentieren, dass sich mithilfe des Konzepts der Kommunikationslatenz, aber auch das Phänomen der Abwehr der Kritik von Antisemitismus als funktionale soziale Tätigkeit beschreiben lässt. Hierzu muss der Begriff der »Latenz« als Oberbegriff für alle Verhaltensmuster gefasst werden, die das Potenzial haben, nicht Antisemitismus, sondern dessen Kritik aus dem öffentlichen Diskurs abzudrängen.

Das von Bergmann und Erb entwickelte Theorem der Kommunikationslatenz geht von dem Umstand aus, dass Antisemitismus nach der Niederlage des Nationalsozialismus, als auf Ausgrenzung, Verfolgung und Vernichtung zielendes politisches Projekt, zwar militärisch besiegt und moralisch diskreditiert worden war, allerdings auch nach 1945 als privates (Massen-)Vorurteil fortexistierte. Dies habe das politische System der jungen westdeutschen Nachkriegsdemokratie vor große Herausforderungen gestellt. Den Al- 
liierten gegenüber musste ein interner Wandel zum Besseren dokumentiert werden, um auf die (Re-)Integration der BRD in die Staatengemeinschaft hoffen zu können. Die Existenz des verbreiteten Judenhasses in der Nachkriegszeit stand diesem Interesse diametral gegenüber (vgl. Bergmann/Erb 1986: 228). Damit verbunden war den Autoren zufolge weiterhin, dass die westdeutsche Demokratie zunächst eine »frei schwebende Konstruktion« (ebd.) darstellte und deshalb ein internes Stabilitätsproblem besaß. Hieraus folgern Bergmann/Erb, dass es in den Nachkriegsjahren zwei Objekte gab, die in besonderer Weise auf Schutz angewiesen waren: zum einen die labilen demokratischen Strukturen selbst, zum anderen das propagierte Bild einer demokratischen Identität der deutschen Gesellschaft, welches die Funktion erfüllte, die politische, wirtschaftliche und kulturelle Reintegration der BRD zu ermöglichen. ${ }^{4}$ Die Frage, wie diese Strukturen und dieses Bild abgesichert werden konnten, beantworten Bergmann und Erb im Anschluss an Niklas Luhmann mit Verweis auf den Begriff der funktionalen Latenz:

»Latenz blockiert Bewusstsein bzw. Kommunikation, wenn diese zur Zerstörung oder Umstrukturierung innerhalb eines Systems führen würden. Das Latente [d.h. der Antisemitismus, M. H.] verschwindet nicht einfach und auch niemals vollständig, es werden vielmehr Strukturen ausgebildet, die regeln, was in welchen Situationen gesagt bzw. verschwiegen, gesehen bzw. übersehen werden muss.«(Luhmann 1980: 86, zitiert nach ebd.: 226)

Der Begriff der funktionalen Laten $z^{5}$ beschreibt demnach im Grunde keinen Zustand, sondern er bezeichnet das Ensemble von strukturierten, zielgerichteten Tätigkeiten: sagen/schweigen, sehen/übersehen. Hinsichtlich des Phänomens Antisemitismus besagt das zweierlei: Erstens, dass die Kommunikation antisemitischer Einstellungen in der postnationalsozialistischen Gesellschaft (nach und nach) aus der Öffentlichkeit abgedrängt worden ist, indem Antisemitismus problematisiert und dem öffentlich Sagbaren Grenzen

$4 \quad$ Lars Rensmann hat darauf hingewiesen, dass in der DDR dieser Strukturschutz vor allem über die Forcierung eines antifaschistischen Selbstverständnisses und der Externalisierung des Antisemitismus auf die kapitalistische BRD gelöst wurde, womit eine Leugnung des Antisemitismus der »Arbeiterklasse«, eine Marginalisierung der Erinnerung an die jüdischen Opfer des Nationalsozialismus und die Etablierung eines antizionistischen Selbstverständnisses einherging (vgl. Rensmann 2004: 219).

5 Funktionale Latenz bezeichnet im Cegensatz zu faktischer Latenz eine Form der »Selektivität«, die nicht aus Kapazitätsgründen eines Systems resultiert, sondern eine zentrale Rolle für dessen Reproduktion spielt (vgl. Bergmann/Erb 1991a: 276). 
auferlegt wurden. Antisemitismus zählt, so die Autoren, zum Kreis der Themen, die mit starken moralischen Konnotationen versehen sind, so dass nur Konsens oder Dissens möglich ist, wobei die Äußerungen einer anti-antisemitischen Meinung positive Achtungsfolgen, die Äußerung von Antisemitismus hingegen negative Achtungsfolgen zeitige (vgl. ebd.: 234). Hier setzen dann Techniken der Vorurteilsrepression an, die die Kommunikation negativer Einstellungen gegenüber Jüd*innen in der Öffentlichkeit sanktionieren (vgl. ebd.: 227, vgl. auch Holz 2007: 50). ${ }^{6}$

Die Abdrängung des Antisemitismus durch die Moralisierung der Öffentlichkeit und eine Begrenzung des Sagbaren stellt Bergmann und Erb zufolge allerdings nur eine Form dar, um Antisemitismus in die Latenz zu drängen. Der Begriff der Kommunikationslatenz besagt zweitens, das zum Zweck des Strukturschutzes Verhaltenstechniken entwickelt werden, die angesichts antisemitischer Handlungen auf Konfliktverleugnung und -relativierung setzen und die Brisanz antisemitischer Kommunikationen durch »Strategien der Konfliktbegrenzung (Bergmann 1997: 34) entschärfen, indem diese überhaupt nicht als Fall oder als zu vernachlässigender Fall von Antisemitismus ausgewiesen werden (Bergmann/Erb 1986: 228). Hiermit korrespondierten Verhaltensweisen, die das "gesellschaftlich funktionale Verbot [Antisemitismus zu kommunizieren, M. H. ] selbst wieder latent (ebd.: 228) halten, was bedeutet, dass sich Definitionen der Situation etablieren, die nahelegen, dass Antisemitismus überhaupt kein relevantes Problem mehr sei: es wird hierbei also "geleugnet, dass es etwas zu leugnen gibt (Bergmann 1988: 228). Entsprechende Argumentationen stellen also die Notwendigkeit von Antisemitismuskritik grundsätzlich in Abrede.

Im Hinblick auf den gesellschaftlichen Umgang mit Antisemitismus nach 1945 ist es in Anschluss an Bergmann und Erb also notwendig, zwischen zwei verschiedenen Formen der kommunikativen Verdrängung von Antisemitismus zu differenzieren: Während im ersten Fall geäußerter Antisemitismus durch dessen Sanktionierung in die Latenz gedrängt wird, dadurch zugleich

In seinem 1998 erscheinen Aufsatz zur »Kommunikationslatenz und Vergangenheitsbewältigung « (1998) ergänzt Bergmann in Anschluss an Jeffrey K. Olick und Daniel Levy, dass Latenzkommunikation sowohl »taboos and prohibitions« wie auch »duties and obligations « umfasse (Olick und Levy 1997, o. S., zitiert nach ebd.: 407). Zu den Verpflichtungen gehörten in der Nachkriegszeit demnach u.a. die Unterstützung Israels, die Wiedergutmachung und Westbindung, das demokratische Wahlrecht, das Propagieren des Konzepts der >streitbaren Demokratie، sowie das Verbot der Rassenlehre sowie der Widerspruch gegen Antisemitismus und Rechtsextremismus (vgl. ebd.: 395). 
aber als existentes Problem erscheint, geht es im zweiten Fall um die Duldung von Antisemitismus und das Abdrängen von anti-antisemitischen Interventionen. Diese zweite Form von Kommunikationslatenz steht zwar nicht notwendigerweise im offenen Gegensatz zu der die Moralisierung der Öffentlichkeit leitenden Idee, dass Antisemitismus ein Problem darstellt. Sie behindert oder relativiert allerdings den praktischen Vollzug der Problematisierung von Antisemitismus. Wird dies berücksichtigt, so wird deutlich, dass die Äußerung von Antisemitismus nicht in jedem Fall mit Achtungsverlust verbunden sein muss, sondern negative Achtungs-Folgen in antisemitischen Konflikten oder im Anschluss daran auch abgeschwächt oder sogar neutralisiert werden können. ${ }^{7}$

Werden die Verhaltensmuster der Abdrängung von Antisemitismus und der Abdrängung von Antisemitismuskritik bezüglich ihrer Wirkungen auf die zu schützenden Strukturen - das demokratische Selbstbild und labile demokratische Verhältnisse - verglichen, so kann nun zwischen beiden ein wesentlicher Unterschied festgestellt werden. Denn das erste Verhaltensmuster - die öffentliche Problematisierung von antisemitischen Handlungen als illegitime Handlungen - untergräbt zwar die Illusion einer absoluten Diskontinuität der deutschen Gesellschaft gegenüber dem Nationalsozialismus, hat allerdings zugleich das Potential eine solche Diskontinuität durch das offene Engagement gegen ideologische Kontinuitäten auch zu bezeugen. Hierbei geht es, mit Marcus Hawel gesprochen, um die »Kultivierung des Bruches mit der Tradition um einer ganz anderen Normalität willen, die in der Zukunft erst als Tradition erscheinen kann« (Hawel 2006: 63). Antisemitische Äußerungen werden hierbei moralisch skandalisiert, um der Normalisierung antidemokratischer Verhaltensweisen in der Öffentlichkeit entgegenzuwirken. Widerspruch gegen Antisemitismus sichert und festigt also demokratische Strukturen insofern, als es demokratiefeindliche Verhaltensweisen als illegitim ausweist. Formen der Konfliktverleugnung und Bagatellisierung stabilisieren demgegenüber zwar kurzfristig funktionale Bilder personaler oder na-

Vor dem Hintergrund dieser Feststellung erscheinen Annahmen über den »immense[n] Stigmatisierungscharakter von Antisemitismusvorwürfen « (Kohlstruck/Ullrich 2014: 22) in ihrer Pauschalität fraglich. So lassen sich auch etliche aktuelle Gegenbeispiele finden, in denen begründete Antisemitismusvorwürfe gegen Politiker*innen oder prominente Persönlichkeiten kaum längerfristige Folgen haben (vgl. Amadeu Antonio Stiftung 2017: 7, 11, 14). Dies gilt auch für Günter Grass, wie sich spätestens in anerkennenden Reden und medialen Beiträgen anlässlich seines fünfundachtzigsten Geburtstags noch im Jahr 2012 selbst zeigte. 
tionaler Unschuld, unterminieren dadurch aber auch demokratischen Strukturen, indem sie zur gesellschaftlichen Normalisierung von Antisemitismus beitragen. ${ }^{8}$ Damit einher geht, dass der von Bergmann und Erb konstatierte gesellschaftliche Druck auf Menschen, die privat antisemitische Meinungen hegen, deutlich gesenkt wird.

Die Unterscheidung zwischen diesen unterschiedlichen Bedeutungen von Kommunikationslatenz wird häufig übersehen. ${ }^{9}$ So wird unter Latenz zumeist der Fortbestand antisemitischer Denk- und Einstellungsmuster auf der Ebene des Privaten bei gleichzeitiger Abwesenheit von antisemitischer Kommunikation in der Öffentlichkeit verstanden (vgl. z.B. Beyer 2008: 19, Heyder et al. 2005: 155). Dadurch wird die Bedeutung des Latenzbegriffs allerdings halbiert. Wird die Herstellung der Latenz des Antisemitismus in seiner Doppelgestalt als Tätigkeit der Blockierung antisemitischer Kommunikationsmuster und als Blockierung anti-antisemitischer Interventionen betrachtet, so erweist sich Latenz als ambivalentes Phänomen. ${ }^{10}$

Die Überlegungen Werner Bergmanns und Rainer Erbs haben vor allem in der quantitativen Sozialforschung einige Resonanz hervorgerufen und ver-

8 Dies sieht Bergmann bezüglich der ersten Nachkriegsjahre freilich anders, insofern er davon ausgeht, dass die offene Problematisierung der personellen Kontinuitäten des Nationalsozialismus und des verbreiteten Antisemitismus in der Nachkriegszeit Demokratie bedrohende Folgen hätte gehabt haben können. Ähnlich wie der Historiker Hermann Lübbe (vgl. Lübbe 1983) argumentiert er, dass die junge Demokratie zu großen Teilen auf den Kooperationswillen ihrer internen Gegner angewiesen gewesen sei und demokratische Strukturen zunächst gefestigt werden mussten, bevor Themen wie Nationalsozialismus und Antisemitismus überhaupt adressiert werden konnten. Cesine Schwan hat demgegenüber in ihrer Studie zum gesellschaftlichen Umgang mit dem Nationalsozialismus in den Nachkriegsjahrzehnten herausgearbeitet, dass die Nicht-Thematisierung des Nationalsozialismus »erhebliche menschliche und politische >Kosten « verursacht« und einen wirklichen demokratischen Wandel nicht nur nicht »begünstigt, sondern beeinträchtigt, z.T. unterminiert hat.«(Schwan 1990: 72, vgl. hierzu auch: Giordano 1990: 36ff.).

9 Ich danke Werner Bergmann für die ausführliche Erläuterung seines theoretischen Ansatzes, ohne die ich mir diese eigentümliche Doppeldeutigkeit der Latenz nicht hätte erschließen können.

10 Die Tendenz zur Nivellierung dieses Unterschieds findet sich bereits bei den Autoren Bergmann und Erb selber, z.B. wenn das Konzept der Kommunikationslatenz als die »Abdrängung von Antisemitismus aus der öffentlichen Kommunikation« definiert wird (vgl. Bergmann/Erb 1991a: 275f.). Diese Tendenz verstärkt sich noch in Werner Bergmanns Habilitationsschrift (Bergmann 1997), wo die Idee der Latenz mit der Idee eines kontinuierlichen gesellschaftlichen Lernfortschritts verbunden wird. 
schiedene quantitative Folgestudien inspiriert (u.a. Bergmann 1991b, Heyder et al. 2012, Beyer, H./Krumpal 2010). In den letzten Jahren haben sich zugleich Stimmen gemehrt, die infrage stellen, ob sich die These von der Kommunikationslatenz angesichts von wachsender antisemitischer Kommunikation in der Öffentlichkeit nach wie vor aufrechterhalten lasse (vgl. Diner 2004, Rensmann 2004: 493, Stender 2010: 12). Die hier vorgeschlagene Lesart des Latenztheorems zeigt jedoch, dass sich dieses auch auf andere Weise verstehen lässt. Zusammenfassend lässt sich sagen, dass die von den Autoren behauptete Notwendigkeit des Strukturschutzes durch zwei gegensätzliche Strategien erfüllt werden kann: der Problematisierung von Antisemitismus auf der einen, seiner Bagatellisierung, Relativierung und Invisibilisierung auf der anderen Seite.

Diese Feststellung ist insofern wichtig, als sie einer verkürzten Vorstellung der Prozesse und Entwicklungen entgegengestellt werden kann, die davon ausgeht, dass in den Jahrzehnten nach 1945 ein kontinuierlich verlaufender kollektiver Lernprozess eingesetzt hätte, der zur weitgehenden Ächtung von Antisemitismus geführt habe. Die historischen Prozesse scheinen demgegenüber, wie bereits Rensmann angemerkt hat, komplexer und widersprüchlicher (vgl. Rensmann 2004: 183). Soll der theoretische Rahmen von Bergmanns Lerntheorie nicht aufgegeben werden, so scheint es deshalb plausibel, nicht von der Existenz eines, sondern von zwei sich parallel entwickelnden und komplementär gestalteten Lernprozessen auszugehen. Anti-antisemitische Lernprozesse zielen demnach darauf, Antisemitismus kämpferisch zu begegnen. Lernprozesse, die auf die Abdrängung von Antisemitismuskritik bezogen sind, zielen demgegenüber darauf, anti-antisemitische Artikulationen in ihrer Wirkung zu neutralisieren. Die Etablierung von Antisemitismus thematisierenden, wie auch diese Thematisierung abwehrenden Argumentationsmustern, sind hierbei gleichermaßen als Bestandteile der historischen Themenkarriere des Antisemitismus und somit auch der bundesdeutschen Kultur zu betrachten. Gesellschaftliches Lernen sollte also nicht auf die emphatische Idee eines rationalen Fortschritts verkürzt werden, wie beispielsweise Werner Bergmann und Max Miller dies tun (vgl. Miller 2006: 280). Stattdessen sollten Lernprozesse als evolutionäre Innovationsprozesse verstanden werden, die auf der praktischen Erprobung von Handlungsmöglichkeiten und »ideologischer Arbeit (Taguieff 1991: 248) beruhen. Wenn entsprechende Handlungsmodelle, die Einzelne als individuelle Lösungen für ihre Probleme entworfen haben, von anderen übernommen werden und sich von den ursprünglichen individuellen Problemkontexten lösen, kann in An- 
schluss an die Wissenssoziologen Peter Berger und Thomas Luckmann auch von deren »Institutionalisierung « gesprochen werden (vgl. Berger/Luckmann 1980: 58ff.).

\section{Zwischenfazit}

Die Rezeption des Ansatzes der Kommunikationslatenz zeigt, dass sich Abwehr nicht nur in psychoanalytischen, sondern auch in soziologischen Termini fassen lässt, wobei sich die metapsychologische Konzeption allerdings als modellbildend erweist. Abwehr wird von den Autoren kommunikationstheoretisch als Form der Latenzkommunikation beschreiben, d.h. als soziale (und nicht als psychologische) Tätigkeit, die keine Funktionen für ein psychisches, sondern ein politisches System erfüllt. Hierzu lässt sich in historischer Perspektive zum einen die Integration und Einbindung des antisemitisch eingestellten Personals in demokratische Strukturen, zum anderen die Stabilisierung eines positiven Außenbildes des Systems zählen, das die Integration der BRD in die Staatengemeinschaft ermöglichte. ${ }^{11}$ Um dies zu erreichen, zielen entsprechende Tätigkeiten auf die Abschwächung der sozialen Wirkung oder sogar das Abdrängen anti-antisemitischer Interventionen. Latenzkommunikation wird hierbei, im Sinne Ludwig Schneiders, also als soziale Tätigkeit gefasst, die als »Teilelement« eines Systems »eine Aufgabe erfüllt, d.h. ein Problem löst, das für dieses [System] in spezifischer Weise bedeutsam ist « (Schneider 2009: 53). Hinsichtlich solcher Tätigkeiten unterscheidet Schneider in Anschluss an Merton zwischen »latenten « und "manifesten Funktionen«, die solchen Tätigkeiten zukommen. Unter latenten Funktionen werden solche funktionalen Tätigkeiten gefasst, deren Funktion denjenigen, die sie vollziehen, »ex definitione unbekannt sind« (ebd.) und vor allem durch sozialwissenschaftliche Beobachter*innen zugeschrieben werden. Demgegenüber kann entsprechenden Tätigkeiten eine manifeste Funktion zugeschrieben werden, wenn sie nicht nur »aus der Perspektive des Beobachters rational erscheint«, sondern sich durch den »subjektiv handlungsleitenden Sinn«

11 Deutlich wird hier, dass der Geltungsanspruch des Theorems der Kommunikationslatenz auf die ersten Nachkriegsahrzehnte begrenzt ist. Vor diesem Hintergrund wäre es notwendig, die theoretischen Überlegungen zu aktualisieren und zu fragen, welche Funktionen die Abwehr der Kritik von Antisemitismus im politischen System heute spielt. 
einer Handlung ursächlich erklären lässt (vgl. ebd.: 58). Da sich bei den Autoren Bergmann und Erb diese Unterscheidung allerdings nicht finden lässt, bleibt das Theorem der Kommunikationslatenz an dieser Stelle unbestimmt. Tätigkeiten, die auf das Abdrängen von Antisemitismuskritik bzw. das latentHalten bestehenden Antisemitismus hinauslaufen, könnten demnach zum einen objektiv über ihre sozialen Wirkungen, zum anderen - in Anschluss an Max Weber - subjektiv als »soziale Handlungen « und damit als solche Handlungen verstanden werden, die "von dem oder den Handelnden gemeinten Sinn nach auf das Verhalten anderer (bezogen) und daran in seinem Ablauf orientiert « (Weber 1921: 1) sind. Die Ansätze, die in den folgenden Kapiteln vorgestellt werden, argumentieren nun genau im letzteren Sinne, wenn sie abwehrende Kommunikationsmuster als soziale Handlungen beschreiben, die auf das Handeln anderer Menschen einwirken und mit bestimmten Formen subjektiven Sinns verbunden sind. Die Ansätze setzen sich in diesem $\mathrm{Zu}$ sammenhang auch mit zwei weiteren Fragen auseinander, die bei Bergmann und Erb weitgehend offen bleiben: Erstens, wie die allgemeine Form solcher Kommunikationsmuster genauer $\mathrm{zu}$ beschreiben ist und zweitens, warum solche Handlungen in interaktiven Zusammenhängen überhaupt erfolgreich sein können.

\subsection{Abwehr als Technik der Neutralisierung}

Mit dem Phänomen der Abwehr setzen sich auch Gresham M. Sykes und David Matzas in ihrem Aufsatz »Techniques of Neutralization. A Theory of Delinquen$c y$ (1957) auseinander. Die dort verhandelten, ursprünglich aus der Kriminologie stammenden, Thesen sollen im Folgenden im Kontext soziologischer Forschung diskutiert werden. Die Autoren nehmen einige Ideen der Metapsychologie auf, um dann aber vor allem die Bedeutung von abwehrenden Sprechhandlungen für das Individuum als psychisches und als soziales Wesen zu bestimmen.

In ihrer Analyse von Rechtfertigungsmustern krimineller und gewalttätiger Jugendlicher weisen die Autoren zunächst die Überlegung zurück, dass sich die Jugendlichen außerhalb des gesellschaftlichen Wertesystems positionieren, innerhalb dessen ihre Handlungen als Normverstöße sanktioniert werden. Stattdessen sei ihr Verhalten zugleich von der Anerkennung dieses Systems, wie auch von dem Versuch bestimmt, seine moralischen Imperative argumentativ zu unterlaufen. Die Besonderheit der Rechtfertigungsmuster 
liege deshalb darin, dass sie als »tangential or glancing blows at the dominant normative system rather than the creation of an opposing ideology« (ebd.: 669) verstanden werden könnten. In ihren Versuchen der Rechtfertigung bezögen sich Delinquenten auf gesellschaftlich verbreitete und anerkannte Argumentationsmuster, um diese für ihre eigenen Zwecke zu erweitern. Aussicht auf Erfolg hätten sie hiermit, da das Normen- und Wertesystem einer Gesellschaft sozial erwünschte und unerwünschte Verhaltensweisen nicht eindeutig definieren könne, sondern sich durch Flexibilität und Deutungsbedürftigkeit auszeichne (vgl. hierzu auch Schneider 2005: 41). Werte und Normen müssen demnach also - da sie interpretationsbedürftig sind - stets auf konkrete Situationen bezogen werden, wodurch sich aber stets die Möglichkeit ergibt, das eigene Handeln anders zu klassifizieren oder einzuordnen, als andere dies tun. Im Fall der »denial of responsibility « (Verleugnung von Verantwortung) könne dies z.B. folgende Form annehmen:

»It may [...] be asserted that delinquent acts are due to forces outside of the individual and beyond his control such as unloving parents, bad companions, or a slum neighborhood. In effect, the delinquent approaches a >billiard ball conception of himself in which he sees himself as helplessly propelled into new situations. «(Ebd.: 667)

Solche und ähnliche Argumentationen liefen dann auf die psychologische wie auch soziale Neutralisierung der Kritik und Sanktionierung normabweichender Handlungen hinaus:

»Disapproval flowing from internalized norms and conforming others in the social environment is neutralized, turned back, or deflected in advance. Social controls that serve to check or inhibit deviant motivational patterns are rendered inoperative, and the individual is freed to engage in delinquency without serious damage to his self image. In this sense, the delinquent both has his cake and eats it too, for he remains committed to the dominant normative system and yet so qualifies its imperatives that violations are sacceptable if not >right. Thus the delinquent represents not a radical opposition to law-abiding society but something more like an apologetic failure, often more sinned against than sinning in his own eyes. We call these justifications of deviant behavior techniques of neutralization [...].«(Ebd.: 666f., Hervorhebung M.H.)

Techniken der Neutralisierung dienen demnach zum einen dazu, der Erkenntnis auszuweichen, dass durch die eigenen Handlungen de facto 
geltende Normen verletzt werden oder wurden. Im Sinne der Metapsychologie können sie demnach auch als Ersatzbildung eines Abwehrprozesses verstanden werden, mit dem ein Konflikt zwischen dem Ich und dem Gewissen gelöst wird. Interessanter ist allerdings der Hinweis der Autoren, dass Abwehrhandlungen auch dazu fungieren, in die soziale Umwelt (»conforming others") einzugreifen. Sie sind hierbei - so die Autoren weiter - nicht allein auf möglichen »self-blame«, sondern auch auf »the blame of others after the act « bezogen (vgl. ebd.: 666). Das heißt, dass durch kreative Deutungen der Situation auch die Chance herabgesetzt wird von relevanten anderen als Normbrecher stigmatisiert zu werden (vgl. hierzu auch Schwarz-Friesel 2012: 362).

Durch den Hinweis, dass dies auch geschehen kann bevor eine deviante Handlung vollzogen wurde, lassen sich nun zwei Befunde der Rassismus und Antisemitismusforschung in die Argumentation von Sykes und Matza integrieren. Denn zum einen passt hierzu der Befund, dass rassistische oder rechtsradikale Akteure die Strategie verfolgen, durch verschiedene Mittel der Selbstpräsentation für die bürgerliche Mitte oder andere Gruppen diskursiv anschlussfähig zu werden, die eigenen gesellschaftspolitischen Oberziele zu verschleiern und soziale Sanktionsmechanismen zu umgehen (vgl. hierzu Schulze 2009: 85ff.). Zum anderen hat Werner Konitzer festgestellt, dass das vermehrte Auftreten von Antisemitismus häufig »durch eine längere Phase öffentlicher Moralkritik« (Konitzer 2005, o. S.) an einer vermeintlich herrschenden und dogmatischen anti-antisemischen politischen Korrektheit vorbereitet wird. Mit Sykes und Matza wäre dies als Variation der Technik der "Condemnation of the Condemners« zu verstehen, die - präventiv verwendet darauf abzielt, Diskursverschiebungen bezüglich der Grenzen des Sagbaren vorzunehmen und dadurch das gesellschaftliche Normen und Wertesystem insgesamt zu verschieben.

Die Argumentation der Autoren verweist insgesamt darauf, dass Abwehrhandlungen dem Impression Management dienen. Hierunter können in Anschluss an den Soziologen Ervin Goffmannn (Sprech-)Handlungen verstanden werden, die darauf abzielen, die Chance zu erhöhen, dass Interaktionspartner"innen einen möglichst positiven oder jedenfalls keinen negativen Eindruck einer Person erhalten bzw. erhalten werden (vgl. Goffman 1967). ${ }^{12}$ In diesem Sinne sind die Ergebnisse von Matza und Sykes 
auch in der Rassismusforschung rezipiert worden. So hat der Kriminologe Maik Dost in einem sozialpsychologischen Experiment festgestellt, dass Menschen, die Opfern von Rassismus beistehen, gegenüber Interviewer* innen keine Neutralisierungstechniken nutzen, während Schaulustige sowohl dazu tendieren, Gewalttaten zu verleugnen oder den Opfern Verantwortung für die erlittene Gewalt zuzuschreiben, um die eigene soziale Reputation gegen ihre mögliche symbolische Beschädigung abzusichern (vgl. Dost 2008: 2073). Im Unterschied zur Metapsychologie bewirken die von Sykes und Matza beschrieben Abwehrhandlungen also nicht nur die Neutralisierung von Gewissenskonflikten, sondern zielen ebenso auf die Stabilisierung der sozialen Identität einer Person in interaktiven Zusammenhängen. Hier wird deutlich, was auch Jovan Byford betont, dass nämlich Abwehrargumentationen aus soziologischer Perspektive sowohl als Formen der "self-justification« wie auch »justification « $\mathrm{zu}$ verstehen sind (Byford 2013, V: 32). Ein geschickter Delinquent zu sein hieße demnach, Techniken der Neutralisierung zu erlernen und eben nicht, sich in direkte Opposition zum gesellschaftlichen Normsystem zu begeben.

\section{Zwischenfazit}

Der Ansatz von Sykes und Matza unterscheidet sich vom Latenztheorem vor allem darin, dass abwehrende Tätigkeiten nicht in ihrer Funktion zur Stabilisierung des politischen Systems, sondern des psychischen Systems und des sozialen Ichs verstanden werden. D.h. entsprechende sprachliche Äußerungen werden den Autoren zufolge von Individuen nicht nur zur Neutralisierung von Gewissenkonflikten benutzt, sondern auch, um mögliche oder tatsächliche Stigmatisierungen argumentativ zu neutralisieren, um die soziale Bande zu nahestehenden Personen nicht zu gefährden. Sie sind somit nicht wie in der Psychoanalyse allein als psychische Vollzüge, nicht wie bei Bergmann als funktionale Tätigkeiten, sondern als soziale Handlungen ver-

Coffman »an image of self delineated in terms of approvable social attributes« (ebd.: 5), dessen Stabilisierung sich in Interaktionszusammenhängen vollzieht. Goffman kommt insgesamt zu dem Ergebnis, dass Selbstbilder in außerordentlichem Maße gegen Irritationen immunisiert sind und das soziale Umfeld für ihre Stabilisierung eine große Rolle spielt: »Whatever his position in society, the person insulates himself by blindnesses, half-truths, illusions, and rationalizations. He makes an radjustment by convincing himself, with the tactful support of his intimate circle, that he is what he wants to be« (ebd.: 43). 
stehbar, die im Sinne Max Webers auf das wahrscheinliche oder tatsächliche Verhalten anderer bezogen und mit einem subjektiven Sinn verbunden sind.

Über die Frage, ob dieser Sinn solcher Äußerungen den delinquenten Jugendlichen bewusst ist oder nicht, stellen die Autoren allerdings keine Hypothesen auf. Im Anschluss an die Metapsychologie ließe sich vermuten, dass dieser Sinn ihnen nicht bewusst ist. Im Hinblick auf ihre soziale Funktionalität könnte allerdings auch angenommen werden, dass die Rechtfertigungsversuche in bewusst strategischer Absicht verwendet werden und die Jugendlichen also »die Effekte dieses [Handlungs-]Musters kennen und durch ihr Verhalten herbeizuführen beabsichtigen« (Schneider 2009: 58, Hinzufügung M.H.). In beiden Fällen lässt sich das Verhalten unter den Weber'schen Handlungsbegriff fassen. Denn Weber verweist darauf, dass der Sinn einer Handlung den Handelnden bewusst sein kann, aber nicht sein muss. Weber unterscheidet zwischen zwei Typen des nicht-bewussten Sinns einer Handlung. Zum einen stellt der Soziologe fest, dass reales Handeln aufgrund von Gewohnheit »in der großen Masse seiner Falle in dumpfer Halbbewußtheit « (Weber 1921: 10) verlaufe. Zum anderen orientiert sich Weber aber auch am Konzept des Unbewussten, wenn er feststellt, dass »vorgeschobene >Motive und >Verdrängungen< (d.h. zunächst: nicht eingestandene Motive) oft genug gerade dem Handelnden selbst den wirklichen Zusammenhang der Ausrichtung seines Handelns« (vgl. ebd.: 4) verhüllten. Dementsprechend könnte es also sein, dass sich die Jugendlichen über den sozialen Zweck ihrer Handlungen täuschen, wodurch dieses Handeln aber nicht aufhört, sinnhaft-strukturiertes Handeln zu sein. Mit Ludwig Schneider lässt sich aus diesem Grunde darauf hinweisen, dass in diesem Fall eine »inhaltliche Differenz von subjektiv bewußtem (und gegebenenfalls durch Befragung ermittelbarem) aber kausal unwirksamem Sinn einerseits und unbewußtem, das Handeln verursachenden Sinn andererseits (Schneider 2008: 46) vorgenommen werden muss.

Des Weiteren stellen die Autoren Überlegungen dazu an, warum Abwehrhandlungen erfolgreich sein können und was also die Gelingensbedingungen von Abwehrhandlungen sind. Demnach käme ihnen in ihrem Bemühen Schuldgefühle oder eine drohende Stigmatisierung abzuwenden, die gesellschaftliche Deutungsbedürftigkeit von Normen und Werten entgegen. Normen und Werte würden durch die neutralisierenden Verhaltenstechniken nicht einfach gebrochen, sondern so umgedeutet, dass das eigene Handeln nicht als (schwerwiegender) Normbruch erscheint. 


\subsection{Abwehr als ideologisches Syndrom}

Eine der Modellierung von sozialen Abwehrhandlungen, die der von Sykes und Matza sehr ähnelt, findet sich nun auch in Theodor W. Adornos Analysen zum Umgang von Erwachsenen mit der nationalsozialistischen Vergangenheit in der Nachkriegszeit. Adorno hatte die Aufgabe, das in den Jahren 1950/51 für das »Gruppenexperiment« in 121 Gruppendiskussionen erhobene Datenmaterial qualitativ auszuwerten (vgl. Pollock 1955: 278ff.). In seiner Analyse wird zunächst deutlich, dass der Nachkriegsdiskurs durch eine Vielzahl von Relativierungsmustern geprägt war, die insbesondere auf die Abwehr der Erinnerung der Judenverfolgung, der kritischen Aufarbeitung des Nationalsozialismus und der Frage nach Schuld und Verantwortung für nationalsozialistische Gewalt abzielten. Auf entsprechende Kommunikationsmuster werde ich im zweiten Teil des Theoriekapitels zu sprechen kommen. An dieser Stelle sollen vor allem einige theoretische Aspekte der Studie diskutiert werden.

Auch wenn Adorno nicht den Anspruch erhebt, das empirische Material psychoanalytisch zu deuten ${ }^{13}$, sondern es dem eigenen Verständnis vor allem um die Rekonstruktion von »Sinnzusammenhängen « und »ideologischen Syndromen« (vgl. ebd.: 283) geht, so kommt er doch nicht umhin Überlegungen zu den psychologischen Motiven von Abwehrverhalten zu formulieren. Hierzu gehört insbesondere, dass dem Autor die Reaktionen in den Gruppen nur erklärlich erschienen, wenn man von der Annahme ausgehe, »daß tatsächlich etwas wie eine latente Erfahrung von der Schuld vorliegt und daß diese Erfahrung verdrängt und rationalisiert wird. Aber sie muß die ÜberIch-Instanzen der meisten Versuchsteilnehmer in irgendeiner Weise belasten.« (ebd.: 280). Allerdings gäbe es auch einen anderen gewichtigen Grund, die Frage nach Schuld und Verantwortung kategorisch von sich zu weisen. Denn der Anerkennung ihrer Relevanz sei der Wunsch entgegengesetzt »die eigene überwertige Identifikation mit dem Kollektiv, zu dem man gehört, in Übereinstimmung zu bringen mit dem Wissen vom Frevel: man leugnet oder verkleinert ihn, um nicht der Möglichkeit jener Identifikation verlustig zu gehen " (ebd.: 281). Wie in der Analyse des sekundären Antisemitismus auch (siehe Kapitel 2.9), wird also der Wunsch nach einer ungestörten Identifikation mit der deutschen Nation als individuelles Motiv von Abwehrhalten betrachtet. An anderer Stelle geht der Autor davon aus, dass ein Hauptmotiv abwehrender Argumentation auch in der Furcht von Parteimitgliedern vor 
politischer Verfolgung und moralischer Diskreditierung bestehe (vgl.: 314$){ }^{14}$ Ohne dass Adorno dies ausdrücklich benennt, finden sich also - wie bei Sykes und Matza auch - in seinen Analysen sowohl Hinweise auf die psychologische wie auch die soziale Funktion von schuldabwehrenden Argumentationsweisen. In letzterer Hinsicht sind diese u.a. auch als Reaktionen auf Tendenzen des öffentlichen Diskurses der Nachkriegszeit beschreibbar, innerhalb dessen die personale Zugehörigkeit zum politischen Apparat des Nationalsozialismus zumindest potenziell ein Problem darstellen konnte. ${ }^{15}$

Nach Adorno sind abwehrende Argumentationen insgesamt als Ausdruck des Versuchs zu verstehen, Fragen nach Schuld und Verantwortung von sich und der nationalen Wir-Gruppe fernzuhalten, ohne hierbei die Motive, die diesem Versuch zugrunde liegen, mit zu kommunizieren. Der Bezug der Teilnehmer*innen des Gruppenexperiments auf Argumentationen und Sprache im Allgemeinen, sei deshalb instrumenteller Natur. Aus diesem Grund seien Argumentationen der Abwehr durch allerlei argumentative Kniffe, »kunstvolle Unlogik« (ebd.: 307, vgl. auch ebd.: 318) und durch »Virtuosität« (ebd.: 310) geprägt. ${ }^{16}$ So würden beispielsweise schwache Argumentationen mit Hilfe von spitzfindigen Unterscheidungen verstärkt, oder es werde mit der »Logik der Wahrscheinlichkeit« verfahren:

»Nach jedem Krieg wird gesagt, die Deutschen sind schuldig gewesen, und das kann doch wohl nicht der Fall sein, sondern jedes Ding hat seine zwei Seiten, und dieser Restbestand eines eingeschliffenen >common senserSchlusses bewirkt dann unausdrücklich die kühne Extrapolation: also sind wir unschuldig.«(Ebd.: 311)

Solche und ähnliche Argumentationen würden Gegenargumenten zwar kaum standhalten, verliehen allerdings der Abwehrhaltung unter Gleichgesinnten

Diese These korrespondiert mit der von Mitscherlichs aufgestellten Überlegung, dass die »Derealisierung der Vergangenheit« u.a. auch aus der Angst vor Bestrafung durch die Alliierten Siegermächte resultiere (vgl. Mitscherlich/Mitscherlich 1977: 46, vgl. auch Brunner 2011: 15). Dass die damit korrespondierende Furcht in weiten Teilen unbegründet war, hat u.a. Norbert Frei in seiner Analyse der Vergangenheitspolitik nach 1945 gezeigt (vgl. Frei 2005: 27ff.). 
den Schein der Legitimität. ${ }^{17}$ Allerdings betont Adorno auch, dass sich Versuchsteilnehmer*innen in der Entwicklung abwehrender Argumentationen »nur in extremen Fällen der ungezügelten Phantasie« (ebd.: 331f.) überließen. Stattdessen nutzten sie "reale Momente, die sie in den Zusammenhang ihrer Zwecke einschmelzen« (ebd.: 332). Hierfür böten sich beispielsweise Verweise auf die Wirksamkeit der nationalsozialistischen Propaganda oder des nationalsozialistischen Terrors an. Diese Argumentationen seien zwar an und für sich nicht als ideologisch zu bezeichnen, würden aber für die Schuldentlastung instrumentalisiert, so dass es zu einem »bewußte[n] oder unbewußte[n] Mißbrauch der Wahrheit« (338f.) komme:

»Motive, denen als solchen Recht zukommt, treten in Zusammenhänge, in denen ihr Wahrheitsgehalt einzig noch die Funktion hat, von begangenem Unrecht abzulenken, Schuld in Unschuld zu nivellieren, und von einem vorgeblich höheren Standpunkt her, der das Subjekt aus seiner tatsächlichen Situation herausnimmt, es von jeglicher bestimmten Verantwortung zu entbinden. Innerhalb der gesellschaftlichen Verflechtung gibt es kaum ein Argument, das nicht, derart herausgebrochen und isoliert, für solche Zwecke verwendbar wäre, wie es denn überhaupt keinen Gedanken gibt, und wäre er an sich noch so wahr, der nicht, hypostasiert und der lebendigen Erfahrung entzogen, in Wahn und Lüge übergehen könnte. [...] Die subtilen Übergänge von wahren Prämissen zu falschen Konklusionen entziehen sich in der Eile des Gesprächs nur allzu leicht dem Gegenargument, die partielle Wahrheit eines Arguments wirbt Vertrauen auch für den Rest und für den wie sehr auch fragwürdigen Zweck.« (Ebd.: 339)

Da entsprechenden Argumentationen also ein funktionaler bzw. instrumenteller Charakter zukäme, verwandelten sich diese zu ideologischen Formationen. ${ }^{18}$ Sie können hierbei auch als Formen des "post-hoc reasoning« (Schwitz-

17 In diesem Sinne argumentiert auch Ralph Giordano, wenn er feststellt, dass Personen, deren Verhalten durch Abwehr bestimmt ist, zwar in der Gruppe selbstsicher auftreten, aber »unter den Bedingungen einer Zweierdiskussion « kaum noch ihren eingenommenen Standpunkt verteidigen könnten (vgl. Giordano 1990: 17).

18 Auch Rolf und Barbara Vogt argumentieren in ihrer Analyse der Coldhagen-Debatte, dass berechtigte Kritik an Formen der wissenschaftlichen Aufarbeitung der nationalsozialistischen Vergangenheit in den Dienst der Abwehr gestellt werden kann (vgl. Vogt/Vogt 1997: 565). Anzumerken ist hier außerdem, dass eine analoge Argumentation auch in der Vorurteilsforschung vertreten wird. So fassen Milbradt und Knappertsbusch Befunde der Rassismusforschung zusammen, die vorurteiliges Handeln nicht 
gebel/Ellis 2016: 27) bezeichnet werden, wobei ein nicht sichtbares Motiv die Suche nach möglichen Argumentationen, die bestimmte Schlussfolgerungen ermöglicht, anleitet.

\section{Zwischenfazit}

Wie sich zeigt, ist die Konzeptualisierung von Schuldabwehr-Argumentationen bei Adorno der Analyse von Rechtfertigungsmustern bei Sykes und Matza in vielerlei Hinsicht ähnlich. Es wird hierbei davon ausgegangen, dass Abwehrargumentationen zur internen Stabilisierung des psychischen Systems beitragen, insofern sie Gewissenskonflikte neutralisieren bzw. dafür sorgen, dass die Idee einer schuldfreien Nation weiterhin ihre Funktion als Identifikationsobjekt übernehmen kann. Was Adorno hierbei allerdings nicht ausdrücklich reflektiert, ist, dass zwar verdrängte Motive das Verhalten der Individuen in den Gruppendiskussionen verstehend erklären können, dass es sich bei diesem Verhalten aber auch um soziale Handlungen handelt, die offenkundig nicht nur darauf zielen, sich selbst, sondern auch relevante Andere von den eigenen Vorstellungen zu überzeugen. Des Weiteren wird Abwehr aber auch von Adorno als soziale Handlung beschrieben, die dazu dient, Gefahren für das soziale Ansehen der in den Nationalsozialismus verstrickten Versuchsteilnehmer abzuwenden.

Während Sykes und Matza zufolge Abwehrhandlungen das gesellschaftlich geltende Normen und Wertesystems auf flüchtige Weise verletzen, beschreibt sie Adorno als missbräuchlichen Umgang mit wahren Gegebenheiten. Diese Beobachtung hat für die Analyse von empirischem Material, in dem sich Abwehr dokumentiert, offenbar wichtige Implikationen. Denn hier dürften sich nicht zufälligerweise auch Argumentation identifizieren lassen, die nicht einfach als falsch $<\mathrm{zu}$ bezeichnen sind. Inwiefern entsprechende Argumente strategisch oder nicht-strategisch eingesetzt werden, kann hierbei aber nicht über einem festen Satz an Kriterien bestimmt werden. Vielmehr ist diesbezüglich im Grunde die konkrete Analyse von Motiven, die Sprachhandlungen zugrunde liegen, notwendig, weil entsprechende Indikatoren alles andere als eindeutig sind. Nach Adorno liegen Indizien hierfür vor, wenn

auf die Frage nach der propositionale Unwahrheit verwendeter Stereotype reduzieren, sondern sie an der rhetorischen Funktionalität von Sprechakten festmachen (vgl. Knappertsbusch/Milbradt 2013: 51f.). 
Argumentationsaufwand äußerst einseitig betrieben wird oder bemerkenswert schwache oder widersprüchliche Argumentationen vertreten werden.

\subsection{Abwehr als Rhetorik}

Bei Bergmann und Erb wurde Abwehr, in Anschluss an Luhmann, als Form der Verhaltenstechnik beschrieben, die u.a. Formen des Sagens annehme. Sykes und Matza bezeichnen Techniken der Neutralisierung als Muster der Rechtfertigung. Und Adorno spricht von Argumentationen der Abwehr. Es zeigt sich also, dass Abwehrhandlungen von diesen Autoren überwiegend als Sprechhandlungen beschrieben werden. Insofern diese eine soziale Funktion erfüllten, wird damit zugleich festgestellt, dass durch entsprechende Äußerungen nicht nur etwas gesagt, sondern auch etwas getan wird: Durch sie werden anti-antisemitische Interventionen aus dem Diskurs abgedrängt (Bergmann/Erb), die sozialen Folgen von Normbrüchen abgewendet (Sykes/Matza) oder von Fragen nach Schuld und Verantwortung abgelenkt (Adorno). Werden Abwehrtätigkeiten auf diese Weise gefasst, so liegt es nahe, sie mit sprechakttheoretischen Überlegungen zu verbinden. Eben dies geschieht im Feld der diskursiven Psychologie. Diese hat das Phänomen der Abwehr in verschiedenen Themenkontexten - z.B. in Debatten über Klimawandel und Sexismus - und in unterschiedlichen Ländern untersucht (vgl. Cohen 2000, Szuchewycz 2000, Seu 2010, Nelson 2013) und beschäftigt sich in besonderem Maße mit der Frage, auf welche Weisen die Abwehr von potentieller oder tatsächlicher Rassismuskritik im alltäglichen Sprechen und Interagieren von Menschen vollzogen wird (vgl. van Dijk 1987, 1992, Barnes et al. 2001, Goodman/Rowe 2014, RojasSosa 2016). Auf entsprechende Befunde wird in Kapitel 2 zurückzukommen sein. Auch hier interessieren mich zunächst vor allem konzeptionelle Überlegungen.

Laut Michael Billig sind unter abwehrenden Argumentationen alle Formen von Argumentationen zu fassen, mithilfe derer die Thematisierung von gesellschaftlichen Problemen absichtsvoll unterbunden wird. Denn, so Billig,

»if we humans possess the rhetorical skills to open up matters for discussion, then so we are equipped with the abilities to close down matters discursively. For every rhetorical gambit to push the debate forward, so there must be analogous rhetorical devices which permit the discursive exploration to be curtailed.«(Billig 1999: 51) 
In jeder Konfliktsituation, in der Rassismus nicht einfach ignoriert, sondern problematisiert wird, seien demnach zwei gegeneinander gerichtete Kräfte am Werk: auf der einen Seite solche, die versuchen, eine bestimmte, auf Rassismus bezogene Themen- oder Problemsetzung diskursiv durchzusetzen und so zu etablieren, so dass sie Resonanz in einem sozialen Raum hervorrufen, auf der anderen Seite solche, die versuchen mit Hilfe diskursiver Mittel entsprechende Diskurse abzubrechen. Sprachliche Äußerungen, die entsprechende Wirkungen entfalten, werden hierbei auch als Rhetoriken bezeichnet. Autor"innen der diskursiven Psychologie zufolge könne jeder kommunikative Akt als rhetorisch bezeichnet werden, wenn dieser dazu entworfen sei, den Ablauf einer Interaktion zu beeinflussen und hierbei ein erwünschtes Ziel zu erreichen (vgl. Seu 2010: 443, Chiang 2010: 278f.). Das Hauptaugenmerk entsprechender Analysen läge, so Goodman und Burke, »on the action orientation of talk [...], that is, what is accomplished in the interaction by what is said rather than focusing on what this tells us about the speakers' internal cognitions" (Goodman/Burke 2010: 328).

Bezüglich der Analyse solcher Rhetoriken wird - im Unterschied zur Metapsychologie - also nicht nach den tiefer liegenden psychischen Prozessen gefragt, die Abwehrrhetoriken erklären lassen. Zugleich kommt die diskursive Psychologie - wie auch Adorno - aber nicht gänzlich ohne die Unterstellung eines Motivs aus, um den Sinn solcher Rhetoriken bezeichnen zu können.

Dies erfolgt relativ pauschal, indem das latente Interesse von rassistischen Akteuren, ihr soziales Ansehen durch Impression Management zu leisten, vorausgesetzt wird. ${ }^{19}$ Vertreter"innen der diskursiven Psychologie betonen, dass es Menschen, die Rhetoriken der Abwehr verwenden, um die Be-

19 Ohne dies auszuführen, scheinen sich die Autor*innen mit dem Begriff der Rhetorik auf eine spezifische Klasse von Sprechakten zu beziehen, die Jürgen Habermas in Anschluss an John Austin als perlokutionäre Sprechakte bezeichnet hat. Diese werden von lokutionären und illokutionären Sprechhandlungen abgegrenzt. Im Falle lokutionärer Akte würde ein*e Akteur*in »etwas sagen«, im Falle illokutionärer Akte werde gehandelt, »indem man etwas sagt« (z.B. wenn eine Behauptung aufgestellt oder einen Befehl gegeben wird) und im Falle perlokutionärer Handlungen schließlich werde etwas bei einer Hörer*in bewirkt »dadurch daß man handelt « (Habermas 2011a: 389). Während sich im Falle illokutionärer Handlungen »die kommunikative Absicht der Sprecher und das von ihnen angestrebte illokutionäre Ziel aus der manifesten Bedeutung des Gesagten « (ebd.) ergebe, zeichneten sich perlokutionäre Akte durch unausgesprochene Absichten aus, die mit einer Sprechhandlung verfolgt werden. Perlokutionäre Sprechakte werden demnach als Formen »verdeckt strategischen Handelns« (ebd: 395) bezeichnet, die der Beeinflussung der Hörer*innen dienen. Wie Habermas 
wältigung moralischer Rechenschaftspflichten gehe. So weist etwa Byford in seiner Analyse der Verleugnung des Antisemitismus in Serbien darauf hin,

»that individuals and groups participate in social interaction as actors in a moral universe, operating within a culturally specific set of rules regarding what is right or wrong, acceptable or unacceptable. Much of everyday interaction [...] is spent accounting for one's beliefs and conduct within the salient framework of values, and negotiating for oneself a credible position within the moral universe. [...] When articulating views on what are widely perceived as controversial matters on race, ethnicity, and religion, speakers go to considerable lengths to deny that they are being prejudiced, presenting their beliefs and actions as compliant with the powerful social norm against public display of intolerance. «(Byford 2013, I: 9)

Am Grunde von Debatten über vermeintliche oder tatsächliche rassistische Verhaltensweisen liege also häufig die Frage, inwiefern sich Individuen in Reaktion auf tatsächliche oder wahrscheinliche Rassismusvorwürfe als moralisch integre und rationale Entitäten darstellen können (vgl. auch Riggs/Due $2010,258)$. Der gefühlte oder tatsächliche Druck, sich in Interaktion als vorurteilsfreies Individuum präsentieren zu müssen, um diskursiv anschlussfähig zu bleiben, sei dabei hoch - so hoch, dass selbst faschistische Akteure nicht umhinkämen zu behaupten, dass sie nicht rassistisch seien (vgl. Billig 1988: 94, Rensmann/Schoeps 2008: 12).

Stanley Cohen weist darauf hin, dass der Umgang mit solchen Rhetoriken individuell erlernt werden müsse. Hierbei stellten sich insbesondere solche Argumentationsmuster als attraktiv dar, die als sozial akzeptabel gelten (vgl. Cohen 2001: 59). Irene Seu geht in Anschluss an Margaret Wetherell und Jonathan Potter davon aus, dass Rhetoriken der Abwehr zu kulturell verfügbaren "Interpretative Repertoires« gehören. Jedes Repertoire beinhalte Abwehrtopoi, die als gesellschaftlich verfügbare Argumentationsmuster zu verstehen seien und es ermöglichten, bestimmte Schlussfolgerungen zu plausibilisieren. Hierbei seien natürlich besonders solche Topoi von Relevanz, mit denen plausibel gemacht werden kann, warum eine Handlung oder eine Person nicht als rassistisch bezeichnet werden solle. Seu betont, dass solche Topoi als Teil des Common Sense und als »ideology in action« einen bedeutsamen Einfluss auf

entsprechende Unterscheidungen für die Untersuchung sozialer Abwehrhandlungen fruchtbar macht, wird im nächsten Kapitel erörtert. 
Diskussionen und Debatten nehmen könnten »because they appear so obvious, not the personal opinion of a particular individual (Seu 2010: 443).

Vor dem Hintergrund solcher Überlegungen wird von Teun A. van Dijk ähnlich wie von Bergmann und Erb - die Überlegung aufgestellt, dass Formen der Abwehr von Rassismus auch politische, kulturelle und soziale Funktionen erfüllen. So werde z.B. durch die Externalisierung von Rassismus auf Rechtsextremist*innen dieser (vermeintlich) leichter bekämpfbar gemacht. Weiterhin würden durch die Inschutznahme von rassistischen Personen, Gruppen und Institutionen reibungsfreie gesellschaftliche Abläufe garantiert und bestehende Machtverhältnisse stabilisiert (vgl. van Dijk 1992: 96). Von besonderer Bedeutung sei hierbei die Etablierung eines "consensus about the ethnic situation« (ebd.: 95), der dazu führe, dass Rassismus nicht mehr als Problem in einer politischen Gemeinschaft erscheint.

\section{Zwischenfazit}

Zusammenfassend lässt sich festhalten, dass sich das Konzept der Abwehr der diskursiven Psychologie gut in die bisherigen Befunde integrieren lässt. Die diskursive Psychologie geht davon aus, dass Abwehrhandlungen rassistisch sprechenden Akteuren dazu dienen, ein positives Außenbild zu stabilisieren. Entsprechende Abwehrhandlungen werden demnach als Rhetoriken verstanden, mit denen Problematisierungen von Rassismus in strategischer Absicht etwas entgegengesetzt wird, um sie in ihren Wirkungen aufzuheben. Dies entspricht insbesondere der in Anschluss an Bergmann und Erb vorgenommenen Bestimmung von Abwehr als Form der Latenzkommunikation, die allerdings hier an einen subjektiven Sinn rückgebunden wird, der entsprechenden Handlungen zugrunde liegt. Erfolgreich sind entsprechende Rhetoriken demnach, wenn ihre Verwendung dazu führt, dass relevante andere im Konfliktfall davon überzeugt werden, dass ein Diskurs über Rassismus oder rassistisches Verhalten unnötig oder irreführend ist. Wenn die diskursive Psychologie darauf verweist, dass sich Individuen in ihrer Suche nach plausiblem Argumentationsmustern aus einem kulturellen Reservoir an Deutungsmustern bedienen können, greifen sie zudem einen Gedanken auf, der im Anschluss an Werner Bergmanns These von kulturellen Lernprozessen als Institutionalisierung von Abwehrhandlungen bezeichnet worden ist. Während sich mit Bergmann aber eher die Entstehung und Auffüllung dieses Reservoirs an Abwehrmustern beschreiben lässt, verweist die diskursive Psy- 
chologie auf den Aneignungsprozess entsprechender Argumentationen, der von Individuen in entsprechenden Konfliktlagen vollzogen wird.

\subsection{Abwehr als Kommunikationspathologie}

Wie ich bereits zeigte, kann mit dem soziologischen Ansatz Bergmanns und Erbs der Abwehr von Antisemitismuskritik die Funktion zugeschriebenen werden, die soziale Integration des politischen Systems nach innen, wie auch seine politische Integration nach außen sicherzustellen. Zudem wurde in der Auseinandersetzung mit sozialpsychologischen Ansätzen deutlich, dass Abwehr ermöglicht, das soziale Außenbild von Personen gegen äußere Gefahren, d.h. zum Beispiel Rassismuskritik, abzusichern, um die sozialen Bindungen zu relevanten anderen nicht zu gefährden. Dass Abwehrhandlungen im Konfliktfall der Stabilisierung von sozialen Beziehungen dienen, betont auch der Soziologe Jürgen Habermas. Im Unterschied zu den bisher genannten Autor*innen, zielt die Habermas'sche Analyse allerdings nicht auf die Analyse der Funktion von Abwehrhandlungen im Kontext manifester, sondern latenter sozialer Konflikte.

Habermas hat in seinen »Überlegungen zur Kommunikationspathologie» (1984) die metapsychologische Bestimmung der Abwehr aufgenommen und im Kontext einer normativ fundierten Kommunikationstheorie weiterentwickelt. $\mathrm{Zu}$ diesem Zweck reformuliert er den psychologischen Mechanismus in kommunikationstheoretischen Termini. Habermas argumentiert, dass allen psychologischen Abwehrmechanismen gemein sei, dass sie als funktionale Kommunikationssperren zu betrachten sind, die angesichts nicht verarbeitbar erscheinender Konfliktlagen zu einer »Unterbrechung der Kommunikation innerhalb des Selbst « führen und »Verständigungsprozesse und damit Heilung (Konfliktlösung) durch Einsicht« (ebd.: 232) ausschließen. Entsprechende Unterbrechungen von Kommunikation beschreibt der Soziologe nun auch hinsichtlich seiner Analyse von Kommunikationsstörungen in Familien, dort also, wo es nicht um intra- sondern um interpersonelle Konflikte und Verständigungsprozesse geht. Hier dienen Kommunikationsabbrüche zur »Stabilisierung eines Handlungszusammenhangs, der zwar mit Konfliktpotential aufgeladen ist, der aber dieses Potential auch bindet und gewissermaßen stillstellt« (ebd.: 253).

Bevor an dieser Stelle auf die Einzelheiten von Habermas' Konzeptualisierung von Kommunikationspathologien eingegangen werden kann, müs- 
sen zunächst in sehr komprimierter Form einige Grundannahmen seines Diskursverständnisses vorgestellt werden. Hierfür ist es sinnvoll, auf einen von Habermas als »typisch « bezeichneten Ausgangspunkt für intersubjektive Verständigungsprozesse zu verweisen:

»Typisch sind Zustände in der Grauzone zwischen Unverständnis und Mißverständnis, beabsichtigter und unfreiwilliger Unwahrhaftigkeit, verschleierter und offener Nicht- Übereinstimmung einerseits, Vorverständigtsein und Verständigung andererseits; in dieser Zone muß Einverständnis aktiv herbeigeführt werden. Verständigung ist also ein Prozeß, der Unverständnis und Mißverständnis, Unwahrhaftigkeit sich und anderen gegenüber, schließlich Nicht-Übereinstimmungen auf der gemeinsamen Basis von Geltungsansprüchen zu überwinden sucht, die auf reziproke Anerkennung ausgelegt sind.«(Ebd: 233)

Was aber ist mit der Basis von Geltungsansprüchen gemeint? Habermas nimmt an, dass es zu den allgemeinen Voraussetzungen kommunikativen Handelns gehöre, dass Sprecher*innen Ausdrücke so wählen, dass sie verständlich sind (Geltungsanspruch der Verständlichkeit), dass Äußerungen so vollzogen werden, dass sie anerkannten Normen entsprechen (Geltungsanspruch der Richtigkeit) und dass Äußerungen so formuliert werden, dass sie die Intention der Sprecher*innen wiedergeben (Geltungsanspruch der Wahrhaftigkeit). Werde eine dieser Voraussetzungen verletzt, müsste dies entweder zu einem Zusammenbrechen oder zu einem Reflexiv-Werden der Kommunikation führen, d.h. die Verletzung müsste also thematisiert oder überwunden werden (vgl. ebd.: 245ff.). In diesem Fall käme es zu einem offenen Konflikt. Vor dem Hintergrund dieser Überlegungen entwickelt Habermas eine interaktionstheoretische Basis für seine Argumentation und unterscheidet zwischen einer »inneren « und einer "äußeren « Organisation von Rede. Während Habermas unter der inneren Organisation der Rede das implizite Erheben von Geltungsansprüchen versteht, die die Grundlage der Rede selbst bilden, werden unter der äußeren Organisation der Rede die sozialen Bedingungen gefasst, im Kontext derer Verständigungsversuche vollzogen werden (vgl. ebd.: 242). Zu Formen der Kommunikationspathologie komme es nun, wenn ein Problemdruck, der in der äußeren Organisation der Rede - z.B. aufgrund latenter Konflikte zwischen den Gesprächspartner*innen - entstehe, an die innere Organisation mit einem »Verzerrungseffekt» weitergegeben werde. Dies könne zu Interaktionspraktiken führen, in denen ein handlungstragender Konsens nur dem Schein nach aufrechterhalten wird 
(vgl. ebd.: 265). Den Ausdruck, den Konfliktvermeidung in intersubjektiver Kommunikation annimmt, bezeichnet Habermas dann als systematisch verzerrte Kommunikation. In dieser werde »die Geltungsbasis sprachlicher Kommunikation unauffällig, d.h. ohne die Konsequenz des Abbruchs der Kommunikation oder des Übergangs zu deklarierten und erlaubtem strategischem Handeln eingeschränkt« (ebd.: 252). $\mathrm{Zu}$ einer entsprechenden Einschränkung komme es, wenn Verständigungsprozesse unter der Annahme des Vorliegens verständigungsorientierten Handelns fortgesetzt würden, obwohl entweder der Geltungsanspruch der Verständlichkeit, der Wahrhaftigkeit oder der Richtigkeit von einer Sprecher*in verletzt worden seien.

Systematisch verzerrte Kommunikation ist demnach als Modus der Kommunikation verstehbar, bei dem interpersonale Bindungen nicht dadurch hergestellt werden, dass Konflikte verständigungsorientiert bearbeitet werden (was Habermas zufolge dann zu einer »rational motivierte Bindung« führt, vgl. Habermas 2011a: 376), sondern dadurch, dass ein Konflikt verschleiert wird. Systematisch verzerrte Kommunikation ermöglicht es hierbei paradoxerweise Kommunikation fortzuführen und befürchtete oder drohende Kommunikationsabbrüche zu vermeiden, indem Kommunikationssperren errichtet werden. Resultat hiervon ist ein bloßer Schein von Konsens, hinter dem sich Dissens verbirgt. Im Unterschied zur Lüge, die als bewusstes und verdeckt strategisches Handeln betrachtet wird, handeln im Falle systematisch verzerrter Kommunikation allerdings »sowohl Sprecherinnen als auch Hörerinnen in der Überzeugung, es liege Verständigungsorientierung vor, während [Habermas zufolge, M.H.] die Sprecherinnen in Wirklichkeit strategische Ziele verfolgten, ohne es selbst zu wissen« (Biskamp 2016: 342). Systematisch verzerrte Kommunikation ist aus diesem Grunde Ausdruck einer »unbewusste[n] Täuschung, die zugleich mit einer Selbsttäuschung einhergeht« (ebd., vgl. auch Gross 2010: 338).

Habermas nennt in diesem Zusammenhang drei Strategien, die im Zuge systematisch verzerrter Kommunikation auftreten. Hierzu gehören erstens Verhaltensweisen, die die genauere Investigation von solchen Geltungsansprüchen verhindern, die einen Konflikt manifest werden lassen. Entsprechende Strategien bestehen z.B. darin, jemandem ins Wort zu fallen, Äußerungen $\mathrm{zu}$ ignorieren, Themenwechsel $\mathrm{zu}$ forcieren oder Themen auf unauffällige Weise zu verschieben (vgl. Habermas 1984: 265f.). Victor Kempf betont diesbezüglich, dass solche Verhaltensweisen den Kooperationswillen der Gesprächspartner*innen voraussetzen, was bedeutet, dass unbewusst strategi- 
schen Sprechakte von der Hörer"in als verständigungsorientierte Sprechakte (miss-)verstanden oder »wohlwollend als wohlwollend « (Kempf 2012: 49) interpretiert werden müssen.

Zweitens identifiziert Habermas sprachliche Techniken, die einen Pseudokonsens herbeiführen, wobei z.B. eine Meinungsverschiedenheit »in der Antwort oder der sonstigen Reaktion als Übereinstimmung formuliert« wird, oder eine »Korrektur der Mitteilung des Senders, durch Beifügen von Worten oder Lauten« (ebd.: 266) vorgenommen wird. Unter Pseudo-Konfirmationen versteht Habermas Formen positiver Anschlusskommunikation, »die ein wesentliches Teilstück der Kommunikation des andern weglassen (əvergessen «) und mit dem Rest als Ganzem operieren« (ebd.: 267). Problematisch sei dies insbesondere, weil sie dadurch die

»komplementäre Sprechhandlung in ihren Ansprüchen auf Verständlichkeit, Wahrhaftigkeit oder Richtigkeit disqualifiziert und ihr zugleich die Anerkennung anderer Geltungsansprüche, die der Sprecher gar nicht erhoben hat, suggestiv unterschiebt. Das mehr oder weniger auffällige Manöver besteht also darin, einen Konsens über Sprecherintentionen und über einen normativen Kontext herzustellen, der den Dissens über das tatsächlich Geäußerte zudeckt.«(Ebd.)

Drittens können Habermas zufolge Sprecher*innen versuchen, andere über ihre Intention durch taktische »Selbstdementierungen « zu täuschen. Um das Bild eines bestehenden Konsenses zu wahren, werden hierbei z.B. weitgehende Zugeständnisse an das Gegenüber gemacht, ohne wirklich von dessen Argumentation überzeugt zu sein (vgl. ebd.: 268).

\section{Diskussion und Zwischenfazit}

Hinsichtlich der Konzeptualisierung des Abwehrbegriffs ähnelt das Theorem systematisch verzerrter Kommunikation insbesondere dem Konzept der Metapsychologie, unterscheidet sich hiervon allerdings auch in mehreren Hinsichten. Es ähnelt ihm insofern, als Abwehr als Form der Bewältigung, aber nicht als lösungsorientierte Bearbeitung von latenten Konflikten verstanden wird. Während die Metapsychologie aber davon ausgeht, dass durch abwehrende Tätigkeiten die Stabilisierung eines psychischen Systems erreicht wird, betont Habermas die Funktion von Abwehrhandlugen für krisenanfällige soziale Beziehungen und Handlungszusammenhänge. Damit zusammen hängt, dass der Metapsychologie zufolge Abwehr mit 
einer (unbewussten) Selbsttäuschung verbunden ist, wohingegen Abwehrhandlungen bei Habermas auch mit der unbewussten Täuschung anderer einhergehen. Des Weiteren wird in der Metapsychologie davon ausgegangen, dass Abwehrprozesse selbst nicht beobachtbar seien. Habermas zufolge sind solche Prozesse als soziale Praktiken allerdings sichtbar und drücken sich in Form der Verhinderung von diskursiven Klärungsprozessen, der Herstellung von Pseudokonsens und taktischen Selbstdementierungen aus. Nicht beobachtbar seien hingegen die individuellen Motive, die solche Handlungen verstehend erklären lassen.

Nun gilt es, Habermas Ansatz in die Antisemitismusforschung zu integrieren, da dies bei ihm selbst nicht geschieht. Es scheint unmittelbar plausibel, dass sich damit z.B. solche Situationen analysieren lassen, in denen Akteur*innen Antisemitismus bei nahestehenden Personen (und damit ein Konfliktpotenzial) wahrnehmen, allerdings ein (unbewusstes) Interesse daran haben, diesen nicht zu thematisieren, weil dies zur Destabilisierung eines Handlungszusammenhanges führen würde, in den beide Akteur*innen eingebunden sind. In diesem Sinne hat z.B. Jean Paul Sartre auf die Strategie des Schweigens im Umgang der französischen Gesellschaft mit Antisemitismus in der Zwischenkriegszeit hingewiesen. Im Schweigen käme demnach durchaus ein gegebenes Problembewusstsein über Antisemitismus zum Ausdruck, allerdings werde der Konflikt mit Antisemit*innen vermieden, da deren Reaktionen gefürchtet würden (vgl. Sartre 1965: 70). In Anschluss an Habermas könnte also gesagt werden, dass hier die Strategie des aktiven Ignorierens von antisemitischen Äußerungen an Stelle einer Auseinandersetzung mit ihnen tritt. Im weiteren Sinn wäre Habermas' Beschreibung von Abwehrhandlungen aber auch für die Analyse von Gruppen relevant, in denen die Thematisierung von Antisemitismus desintegrative Folgen haben könnte. Diese Überlegung wird im nächsten Kapitel in der Diskussion einer Antisemitismusstudie der Psychoanalytikerin Birgit Rommelspacher aufgenommen werden.

Zugleich kann das Konzept systematisch verzerrter Kommunikation auch für die Analyse manifester Konflikte zwischen solchen Menschen, die antisemitische Verhaltensweisen bestimmter Akteur*innen verteidigen und solchen, die diese problematisieren, genutzt werden. Während in diesen die zuletzt Genannten versuchen zu zeigen, dass ein Distanzierungsbedarf vorliegt, versuchen die Erstgenannten, diesen in Abrede zu stellen. Folgt man Habermas' Überlegungen, müssten auch hier unauffällige Formen von Verletzungen der Voraussetzungen kommunikativen Handelns $\mathrm{zu}$ beobachten seien. Allerdings wären die Abwehrtechniken teilweise andere als die von Habermas 
beschriebenen. So wäre in solchen Kontexten z.B. die Strategie der taktischen Selbstdementierung eine sinnlose Verhaltensweise. Demgegenüber könnte es aber auch zu den oben beschrieben Formen von Gesprächsverhalten (forcierte Themenwechsel, ins Wort fallen, Themenverschiebung) kommen, die die genauere Investigation von Geltungsansprüchen verhindern, die mit anti-antisemitischen Interventionen verbunden sind. Drittens wäre es von Habermas ausgehend naheliegend, anzunehmen, dass in solchen Kontexten solidarisierende Personen sowohl versuchen, einen Pseudokonsens mit den problematisieren Akteur*innen (z.B. durch das Weglassen bestimmter Äußerungen in ihrer Rezeption) wie auch einen Pseudodissens mit Antisemitismuskritiker*innen herzustellen, indem diesen skandalisierbare Ausdrücke zugeschrieben werden, die diese eigentlich gar nicht geäußert haben. Die Stabilisierung eines Handlungszusammenhangs durch systematisch verzerrte Kommunikation ginge hierbei mit der forcierten Destabilisierung eines anderen Handlungszusammenhangs (d.h. mit einem rationalisierten Gesprächsabbruch mit Kritiker*innen von Antisemitismus) einher. Eine solche Strategie kann in Anschluss an Max Miller als eine »Forcierung von Dissens « bezeichnet werden, die die Verständigung über tatsächliche Meinungsdifferenzen systematisch blockiert (vgl. Miller 2006: 237f.).

Abschließend ist noch darauf hinzuweisen, dass das Konzept systematisch verzerrter Kommunikation in den letzten Jahren von verschiedenen Autor"innen aufgegriffen und kritisch gewürdigt worden ist (vgl. Strecker 2006, Kempf 2012, Biskamp 2016). Von den verschiedenen Argumenten, die in diesem Kontext entwickelt wurden, sind hier letztlich zwei Aspekte von Relevanz. Erstens weist Floris Biskamp darauf hin, dass die Annahme, dass systematisch verzerrte Kommunikation nur dann vorliege, wenn die Geltungsbasis der Rede aufgrund einer unbewussten Handlungsorientierung verletzt werde, problematisch sei, da sie sich kaum empirisch überprüfen lasse und zudem der Effekt dieses strategischen Handelns - ob bewusst oder unbewusst - der gleiche sei. Biskamp plädiert deshalb in Anschluss an David Strecker für eine Ausweitung des Begriffs, sowohl auf Formen des bewussten als auch unbewussten strategischen Handelns (vgl. ebd.: $350 \mathrm{ff}$.). Dementsprechend kann z.B. davon ausgegangen werden, dass mit dem Ignorieren von antisemitischen Äußerungen in Gruppen auch bewusst strategische Erwägungen verbunden sind, z.B. wenn Anlass zur Vermutung besteht, dass durch einen offenen Konflikt die Reputation der Gruppe beschädigt werden könnte oder wenn gruppeninterne Solidarisierungszwänge existieren, denen Akteur*in- 
nen nachkommen, um den eigenen Status innerhalb der Gruppe nicht zu gefährden.

Zweitens weist Kempf darauf hin, dass systematisch verzerrte Kommunikation zu einer Prägung kultureller Wissenssysteme führen kann, wenn sie in einer Gemeinschaft wiederholt zur Stabilisierung von krisenanfälligen Sozialzusammenhängen genutzt wird. In diesem Fall würden latent bestehende Konflikte aber nicht nur durch die oben beschriebenen Formen von Verhaltensmustern in (bewusst oder unbewusst) strategischer Absicht überspielt. Komplementär bildeten sich auch »ideologische Konsensstrukturen« (Kempf 2012: 58) - z.B. die Vorstellung einer sozial gerechten Welt - durch welche die konfliktbeladenen Sozialzusammenhänge zusätzlich gegen Kritik abgesichert würden. Kempf geht diesbezüglich davon aus, dass entsprechende Deutungsmuster auch das Alltagswissen strukturieren, wodurch es Menschen erschwert werde, bestehende Konflikte überhaupt wahrnehmen zu können. Denn Argumentationen, die diese sichtbar machen könnten, seien im Laufe einer Konfliktgeschichte aus der »kollektiven Intelligibilität der Kommunizierenden« (vgl. ebd.: 44) systematisch ausgeschlossen worden. In Anschluss an Biskamp lässt sich dieses Phänomen auch unter dem Begriff "systematisch verzerrter Kommunikationsbedingungen« (Biskamp 2016: 391) fassen, deren Existenz dazu führt, dass auch unvoreingenommene und nicht-strategisch Handelnde ideologische Konsensstrukturen internalisieren und reproduzieren. Unter diesen Voraussetzungen kann Kempf gefolgt werden, wenn er davon ausgeht, dass

»noch derjenige, der in aufwändigen Prozessen seine unbewussten Motive aufdecken könnte [...] vor dem unhintergehbaren Hintergrund historischer a priori, etablierter Desymbolisierungen und fraglos anerkannter, ideologischer Konsensstrukturen im Fahrwasser systematisch verzerrter Kommunikation festgehalten [wird]« (ebd.: 58).

Was diese Überlegung für die Antisemitismusforschung bedeutet, kann deutlich gemacht werden, wenn an den Befund von Bergmann und Erb erinnert wird, dass im postnationalsozialistischen Deutschland der Antisemitismus als Problem nicht nur durch wiederkehrende Bagatellisierungs- und Relativierungsstrategien, sondern phasenweise auch als Problem durch das populäre Deutungsmuster entsorgt wurde, dass es überhaupt keinen Antisemitismus mehr gäbe. Bergmann und Erb zufolge hatte diese zweifellos eine funktionale Bedeutung für das politische System der Bundesrepublik. Es könnte allerdings auch als eine gewachsene ideologische Konsensstruktur bezeichnet 
werden, die als Bestandteil der Lebenswelt, d.h. als »unproblematische [...], gemeinsam als garantiert unterstellte [...] Hintergrundüberzeugung« (Habermas 2011b: 191), im Lauf der Zeit die Wahrscheinlichkeit herabsetzt, dass sich auch unvoreingenommene Menschen für die Relevanz von Antisemitismus als soziales Problem sensibilisieren. Im Fazit zu Kapitel 2 werde ich diesbezüglich argumentieren, dass prinzipiell alle Formen von Abwehrargumentationen die Etablierung entsprechender Konsensstrukturen bedingen können. Solche Überlegungen haben aber auch für die Analyse von abwehrendem Verhalten, das sich im Kontext von antisemitischen Konflikten dokumentiert, weitreichende Implikationen. Denn offenbar wäre es verkürzt, davon auszugehen, dass die Deutungsmuster, die auf die Dethematisierung von Antisemitismus hinauslaufen, stets als Resultat des (un-)bewusst strategischen Handelns einzelner Individuen zu betrachten sind. Vielmehr kann davon ausgegangen werden, dass Individuen aufgrund ihrer Situierung in einer von Abwehr mitgeprägten Kultur auch solche Deutungsmuster in den Debatten verwenden, die durch ihre strategische Verwendung zwar historisch geprägt worden sind, heute aber zum Bestandteil eines individuell kaum bewussten, aber habituell tradierten »konjunktiven Wissens « gehören, das aus einer ähnlichen kulturellen Prägung resultiert (vgl. Mannheim 1980: 217ff.).

\subsection{Abwehr in Familienkonstellationen}

In der Studie »Schuldlos schuldig« hat die Psychoanalytikerin Birgit Rommelspacher den Umgang junger, nicht-jüdischer, deutscher Frauen mit dem Nationalsozialismus untersucht (vgl. Rommelspacher 1995). In der theoretischen Kontextualisierung ihrer empirischen Ergebnisse geht die Autorin hierbei von zwei einander bedingenden Konfliktlagen aus, die sich, wie weiter unten argumentiert wird, sehr gut auf die Grundannahme des Konzepts systematisch verzerrter Kommunikation beziehen lassen, dass diese durch den Druck in der äußeren Organisation von Rede entstehe. Demnach bestehe erstens ein Konflikt zwischen den öffentlichen Erinnerungsweisen des Nationalsozialismus und den Erinnerungsweisen der Eltern. Während in der Öffentlichkeit über den Nationalsozialismus gesprochen werde, würde in den Familien über die damit verbundenen Verbrechen und die eigenen Verstrickungen zumeist geschwiegen. Diese widersprüchliche Konstellation bedinge nun eine zweite Konfliktlage, in der sich die von Rommelspacher befragten Frauen befänden. So führe die öffentliche Thematisierung des Nationalsozialismus u.a. 
zu einem Misstrauen gegenüber den Eltern und Großeltern und produziere damit einen latenten Konflikt (vgl. ebd.: 16). Dies könne dazu führen, dass die Kinder der Familie aktiv Fragen über die Vergangenheit stellten, wenngleich diese häufig »allgemein und stereotyp« seien (ebd.: 82), weshalb sie von Rommelspacher als Ausdruck des Wunsches verstanden werden, "gar nicht so genau hinschauen zu wollen« (ebd.: 83). Die Familienangehörigen reagierten aber zumeist ausweichend. Rommelspacher deutet dies als Ausdruck des Interesses der Eltern und Großeltern nichts zu sagen, was sich negativ auf ihr Außenbild auswirken könne (ebd.: 84).

Eine Möglichkeit der Thematisierung der Vergangenheit durch die Kinder zu begegnen sei das Schweigen. Dieses Schweigen sei allerdings bloßes Schweigen, da hiermit zugleich zum Ausdruck gebracht werde, dass es durchaus etwas zu erzählen und zu sagen gäbe. Rommelspacher bezeichnet es deshalb auch als ein »beredtes Schweigen« (ebd.: 67, vgl. auch Schwan 1997: 108), das verschiedene Formen annehme. So könnten Fragen z.B. ignoriert oder übergangen, oder die stereotype Antwort gegeben werden, dass man »nichts mitgekriegt « habe (Rommelspacher 1995: 71). Oder aber es werde über die Judenvernichtung gesprochen und dabei zugleich wichtiges nicht gesagt, z.B. wenn das »Geschehen so teilnahmslos erzählt [wird], daß der Inhalt dabei völlig entwertet wird« (ebd.: 72). Des Weiteren könnten auf Fragen bezüglich des Nationalsozialismus auch Antworten gegeben werden, die gar nicht auf die gestellten Fragen eingingen, sondern stattdessen z.B. »Kriegsgeschichten« zum Thema machten (vgl. ebd.: 12, vgl. auch Welzer et al. 2002: 81ff.).

Die größte Aufmerksamkeit widmet Rommelspacher allerdings den typischen Verhaltensweisen der Kinder. Sie stellt hierzu fest, dass den gegenläufigen Tendenzen der Erinnerung in Öffentlichkeit und Familie zwei kontrastierende Formen des Umgangs mit dem Nationalsozialismus auf Seiten der Töchter entsprächen. Entweder werde sich von den Eltern und Großeltern oder einer deutschen Kollektividentität kategorisch abgegrenzt. Dies führe zu einem offenen Bruch mit der Familie oder zu moralistischen Anklagen (vgl. Rommelspacher 1995: 98ff). Oder es komme zu einer forcierten Identifikation mit der Familie, wobei die öffentliche Thematisierung des Nationalsozialismus entwertet werde. Der Konflikt zwischen der öffentlichen und privaten Erinnerung werde demnach entweder zugunsten der einen oder anderen Seite aufgelöst, nicht aber bearbeitet. Rommelspacher betrachtet deshalb beides, sowohl die kategorische Abgrenzung als auch die Identifikation, als zwei unterschiedliche Gesichter der Abwehr. Beides ziele letztlich darauf, sich die mit dem nationalsozialistischen Erbe verbundenen Gefühle, Unsicherheiten und 
Konflikte vom Leib zu halten (vgl. ebd.: 16ff.). ${ }^{20}$ Für den Fortgang der hier zu entwickelnden Argumentation ist nun insbesondere das Phänomen der forcierten Identifikation von Interesse.

Rommelspacher geht diesbezüglich davon aus, dass im Zuge der Sozialisation eine »Übernahme der Selbstbilder der Eltern durch die Kinder« (ebd.: 83) stattfände und die Eltern als Identifikationsfläche für die Bildung der eigenen Ich-Identität dienten. Kinder hätten also »ein Interesse daran, ihre Eltern in einem möglichst guten Licht zu sehen, um von deren Kraft und Macht zu zehren.« (Ebd.: 84) Auf der anderen Seite gewährleiste die Stabilisierung eines positiven Bildes der Eltern nicht nur die eigene Selbstachtung, sondern auch den konfliktfreien Kontakt mit den Eltern. Für viele Kinder bestehe nun das Problem darin, dass das Abblocken von Fragen durch die Eltern den Verdacht nähre, »daß es etwas Schlimmes sein muß, das sie zu verbergen haben« (ebd.: 90). Zugleich fürchteten sie aber auch, dass das Aufdecken dieses Verborgenen die Beziehung zu den Eltern grundsätzlich gefährden könne, d.h. sowohl praktisch (Beziehungsabbruch), wie auch symbolisch (Verlust der Vorbilder). Deshalb nähmen nun viele Kinder gegenüber den Eltern eine "Schonhaltung« (ebd.: 85) ein. Aus dieser Gemengelage von Motiven resultiere dann laut Rommelspacher »ein heimliches Bündnis zwischen Eltern und Kindern [...], um das gemeinsam aufgebaute Bild von Eltern und Großeltern nicht zu zerstören, die Familienlegende abzustützen und sie auch nach außen hin zu verteidigen« (ebd.). Abwehr nähme dann einen kooperativen Charakter an, und die Generationen arbeiteten häufig im Sinne einer gemeinsamen Arbeit an einer »Neutralisierung« der beunruhigenden Aspekte der nationalsozialistischen Vergangenheit. Die Abwehrargumentationen der Eltern würden hierbei von den Kindern mitunter eins-zu-eins übernommen (vgl. hierzu auch Rosenthal 1992: 476). Hierzu gehören insbesondere Entlastungsnarrative, (sekundärantisemitische) Argumentationen, die auf die Nivellierung von Schuldfragen hinauslaufen sowie die Bagatellisierung und die räumliche Distanzierung von Antisemitismus.

\section{Zwischenfazit}

Rommelspacher analysiert die Abwehr, sich in deutschen Familien mit dem nationalsozialistischen Erbe kritisch auseinanderzusetzen und den Umstand,

20 Zu sehr ähnlichen Ergebnissen kommen auch Vogt/Vogt in ihren empirischen Analysen zum Verlauf der Coldhagen-Debatte (vgl. Vogt/Vogt 1997: 564f.). 
dass die schulische und mediale Erinnerung an den Judenmord Ursachen dafür zu seien scheinen, dass sich bestimmte Abwehrstrategien in den Familien ausbilden. ${ }^{21}$ Bei genauerer Betrachtung der Analysen Rommelspachers zeigt sich zudem, dass es ihr gelingt, mehrere der bereits diskutierten Theorieansätze miteinander in Beziehung zu setzen. Im Sinne sozialpsychologischer Überlegungen stellt Rommelspacher erstens fest, dass das Verhalten der Eltern als Versuch zu verstehen ist, ihr gutes Ansehen gegenüber den Kindern zu wahren. Komplementär dazu geht sie auf Basis metapsychologischer Überlegungen zweitens davon aus, dass das Abwehrverhalten der Kinder als der Versuch zu verstehen ist, das für ihre Ich-Identität funktionale Bild der moralischen Eltern gegen Irritationen zu schützen. Im Sinne Habermas' integriert sie aber drittens auch die Überlegung, dass Abwehrverhalten für die Stabilisierung eines krisenanfälligen Handlungszusammenhangs zwischen Großeltern, Eltern und Kindern von Bedeutung ist. Vor dem Hintergrund der von Habermas angestellten Überlegungen zu Kommunikationspathologien scheint es angemessen, das beobachtete Verhalten der Eltern und Großeltern (insbesondere das Ignorieren von Fragen und forcierte Themenwechsel) als Ausdruck der von Habermas beschriebenen Strategie zu verstehen, einen gefährdeten Konsens abzuschirmen, indem verhindert wird, dass »hinreichend genau identifiziert werden kann« (Habermas 1984: 265), was diesen Konsens gefährdet. Dies lässt sich als Resultat eines durch die öffentliche Erinnerung des Nationalsozialismus induzierten, latenten Konflikts verstehen, der dazu führt, dass in der äußeren Organisation des Familiengesprächs ein Druck entsteht, der dann an die innere Organisation der Rede weitergegeben und hier durch systematisch verzerrte Kommunikation bewältigt wird. Im Sinne Kempfs ist weiterhin zu ergänzen, dass im Zuge des generationenübergreifenden Gesprächs auch ideologische Konsensstrukturen entstehen (`sie haben nichts gewusst<, >sie waren Opfer des Krieges` usw.), die dazu führen, dass sich die gemeinsam entwickelte Familienlegende als Fakt etabliert, der nicht weiter hinterfragt wird.

21 Zu diesem Schluss kommen auch Harald Welzer und Kolleg*innen, in ihrer Studie zum Umgang der ersten, zweiten und dritten Ceneration. Die Autor*innen argumentieren, dass es gerade die »gelungene Aufarbeitung über die Verbrechen der Vergangenheit zu sein [scheint, M. H. ], die bei den Kindern und Enkeln das Bedürfnis erzeugt, die Eltern und Großeltern im nationalsozialistischen Grauen so zu platzieren, dass von diesem Grauen kein Schatten auf sie fällt« (Welzer et al. 2002: 13). 


\subsection{Diskussion der theoretischen Befunde und Zwischenfazit}

In diesem Kapitel wurden einschlägige Theorieansätze zur Konzeptualisierung von Abwehr aus dem Bereich der Antisemitismusforschung und der Erforschung anderer Gewalt-Phänomene vorgestellt, die auf einem Kontinuum zwischen metapsychologischen und sozialwissenschaftlichen Ansätzen zu verorten sind. Abwehr metapsychologisch zu fassen bedeutet, einen Fokus auf psychologische Prozesse zu legen. Sozialwissenschaftliche Ansätze betrachten Abwehr demgegenüber (auch) als Form sozialer Praxis, kommen allerdings - abgesehen vom Theorem der Kommunikationslatenz selbst nicht ohne subjekttheoretische oder psychoanalytische Überlegungen aus. Abschließend werden hier nun die wesentlichen Ähnlichkeiten und Unterschiede der verschiedenen Konzepte zusammengefasst. Damit wird der begriffliche Rahmen umrissen, der dazu dienen kann, Reaktionen auf die mögliche oder tatsächliche Problematisierung von Antisemitismus theoriegeleitet untersuchbar zu machen.

\section{Konzeptualisierungen von Abwehr}

In metapsychologischen Ansätzen wird argumentiert, dass unter Abwehr ein unbewusster, d.h. nicht-sichtbarer Prozess zu verstehen ist, mit dem die psychische Instanz des Ichs auf äußere Bedrohungen oder innere Konflikte reagiert, um diese psychisch zu bewältigen, ohne sie aber wirklich zu bearbeiten oder zu lösen. Abwehrmechanismen gewährleisten damit die Konstanz, Selbstachtung und Integrität des Individuums und führen zur Etablierung von rationalisierungsbedürftigen Ersatzbildungen, in denen der Abwehrkonflikt nur noch in entstellter Weise aufscheint. Im Anschluss an die Antisemitismusforschung wurde gezeigt, dass dieser Begriff sowohl für die Analyse des Antisemitismus selbst wie auch für die Analyse von Umgangsweisen mit der nationalsozialistischen Vergangenheit Deutschlands und dem damit zusammenhängenden Schuldabwehrantisemitismus fruchtbar gemacht worden ist. Als für die Abwehrprozesse ursächlich werden hierbei verdrängte Aggressionen, Schuld- und Schamgefühle und Identitätskrisen gezählt. Zudem wurde in Anschluss an die Forschung zum Hostile Media Phenomenon festgestellt, dass Abwehrverhalten auch als Ausdruck des Versuchs verstanden werden kann, angesichts der öffentlichen Problematisierung negativer Einstellungen gegenüber Jüd*innen, diese gegen ihre Infragestellung zu immunisieren. 
Demgegenüber wurde gezeigt, dass sich mit den Theorem der Kommunikationslatenz Abwehr als Bündel sozialer Tätigkeiten verstehen lässt, das auf anti-antisemitische Thematisierungen bezogen ist und historisch die Funktion erfüllte, durch das Abdrängen oder Abschwächen von Antisemitismuskritik zwei Cluster von Handlungszusammenhängen gegen Irritationen zu stabilisieren: Erstens spielte Latenzkommunikation eine bedeutsame Rolle im Sinne einer postnationalsozialistischen Sozialintegration, um das in weiten Teilen antisemitisch eingestellte Personal des Nationalsozialismus in die neuen demokratischen Strukturen integrieren zu können. Zweitens gewährleistete sie durch die Stabilisierung eines positiven Wir-Bildes die politische, kulturelle und wirtschaftliche Integration Deutschlands auf internationaler Ebene. Es wurde diesbezüglich argumentiert, dass diese Konzeptualisierung der psychoanalytischen Modellierung von Abwehr zwar ähnlich ist, aufgrund der systemtheoretischen Verortung aber letztlich ohne die Annahme von subjektiven Motiven auskommt, die Abwehrhandlungen zugrundliegen.

Mit Hilfe des zuletzt diskutieren Habermasschen Theorems $\mathrm{zu}$ Kommunikationspathologien konnte demgegenüber herausgearbeitet werden, dass Abwehr aus Perspektive einer verstehenden Soziologie als Form der sozialen Handlung zu bestimmen ist, mit der angesichts latenter Konflikte versucht wird, die Reproduktion und Konstanz krisenanfälliger Handlungszusammenhänge zu gewährleisten. Statt von Abwehrmechanismen spricht Habermas allerdings von Formen des unauffälligen Abbruchs von Verständigungsprozessen. Auch von Habermas wird Abwehrverhalten nicht als lösungsorientierte Bearbeitung von sozialen Konflikten verstanden, sondern als Form der unbewussten Selbsttäuschung, die mit der unbewussten Täuschung anderer einhergeht. In Anschluss an Biskamp wurde argumentiert, dass entsprechende Handlungen allerdings auch als Versuch der bewussten Täuschung anderer verstanden werden können. Die Wirkung einer sozialen Abwehrhandlung besteht also nicht in der Etablierung einer psychologischen Ersatzbildung, die für das Ich akzeptabel ist (siehe S. 42), sondern in der Etablierung eines für andere geltenden Eindrucks, dass sie sich in einem akzeptablen (d.h. nicht mit Konflikten aufgeladenen) Verhältnis zur handelnden Akteur*in befinden.

Die Zugänge von Sykes und Matza, Adorno, der diskursiven Psychologie und Birgit Rommelspacher bewegen sich dann zwischen dem metapsychologischen Konzept der Abwehr und dem soziologischen Konzept von Jürgen Habermas. Zu unterscheiden sind hierbei Ansätze, die den funktionalen Charakter von Abwehrhandlungen für gewaltvoll Handelnde betonen und An- 
sätze, die die Funktion der Abwehr für diejenigen beschreiben, die Konflikten mit antisemitisch Handelnden oder Personen mit einer antisemitischen Vergangenheit aus dem Wege gehen wollen. $\mathrm{Zu}$ den letzten gehört der Ansatz Birgit Rommelspachers, die davon ausgeht, das abwehrende Handlungen dem Zweck dienen, innerfamiliären Konflikten auszuweichen. Demgegenüber schreiben Adorno, Sykes und Matza und die diskursive Psychologie Abwehrhandlungen eine rhetorische Funktion zu, insofern sie angesichts der Problematisierung des eigenen (aktuellen oder vergangenen) Verhaltens als rassistisch, antisemitisch oder gewaltvoll den Zweck erfüllt, die eigene soziale Integration durch Impression Management sicherzustellen.

Ließe sich daraus folgern, dass es angemessen ist Abwehr als soziale Handlung zur Stabilisierung von Handlungszusammenhängen der Abwehr als inneren Prozess zur Stabilisierung des Ichs gegenüberzustellen? Wenn die metapsychologischen Überlegungen berücksichtigt werden, muss dies verneint werden. Denn was für die Skizzierung der Modellierungsmöglichkeiten des Abwehrbegriffs als soziale Kategorie noch fehlt, ist zum einen die Einbindung der Beobachtung Adornos sowie Sykes' und Matzas, dass Abwehr-Argumentationen auch in von Interaktion geprägten Situationen für die Neutralisierung von Gewissenskonflikten instrumentalisiert werden. Zum anderen betonen Adorno und Rommelspacher, dass Abwehrhandlungen in interaktiven Zusammenhängen (d.h. in Gruppendiskussionen und Familiengesprächen) auch dazu dienen können, relevante psychische Identifikationsobjekte (bei Adorno die Nation, bei Rommelspacher die Eltern) diskursiv gegen Infragestellungen zu verteidigen. In diesem Fall scheint es angemessen davon auszugehen, dass Individuen in bestimmten Situationen nach sozialer Anerkennung für die eigenen Formen der Bewältigungsversuche psychischer Konflikte streben bzw. versuchen, den gefühlten sozialen Druck herabzusetzen, sich mit innerpsychischen Konflikten auseinandersetzen zu müssen.

\section{Motive abwehrenden Handelns}

Bezüglich der Motive abwehrenden Verhaltens legen Ergebnisse der Metapsychologie nahe, dass die Wahl von Argumentationsmustern, die der Abwehr dienen, nicht zufällig erfolgt, da in entsprechenden Argumentationen das latente Motiv der Abwehr auf entstellte Weise zum Ausdruck gebracht wird. Allerdings ist es eine offene Frage, ob dies auch dann der Fall ist, wenn Abwehrargumentationen vor allem soziale und nicht psychologische Wirkungen entfalten sollen. In Anschluss an Alfred Schütz scheint es deshalb sinnvoll 
bezüglich der theoretischen Bestimmung von Abwehrhandlungen als soziale Handlungen zwischen $\mathrm{Um}-\mathrm{Zu}$-Motiven und unterschiedlich tiefgreifend in die Psyche eingebundenen »Weil-Motiven« von Abwehrhandlungen zu unterscheiden. Während Schütz mit »Um-Zu-Motiven« den unmittelbaren Zweck bestimmt, der mit dem Entwurf einer Handlung verbunden ist, zielt die Feststellung von »Weil-Motiven « auf die Frage, aus welchen lebensweltbezogenen Gründen, d.h. warum diese Handlung vollzogen wird (Schütz 1974: 122f.). ${ }^{22}$

Bezüglich des Vorliegens von Weil-Motiven, die der Abwehr von Thematisierungen von Gewalt zugrunde liegen, kann in Anschluss an die diskutierten Autor"innen zusammenfassend zu folgenden Schlüssen gekommen werden. $\mathrm{Zu}$ sozialen Abwehrhandlungen kommt es demnach u.a. dann, wenn

- Individuen damit versuchen auf den Nationalsozialismus bezogene (entlehnte) Schuldgefühle zu bewältigen und im Vollzug dieses Versuchs nach der Anerkennung anderer streben,

- antisemitische Einstellungen oder das für Identitätsbildungsprozesse wichtige Konzept der Nation gegen Infragestellungen in Schutz genommen werden sollen,

- ein (politisches) Interesse an der kontrafaktischen Stabilisierung des Außenbildes einer von Antisemitismus bereinigten Gemeinschaft besteht,

- ein Interesse besteht, antisemitische Personen oder Gruppierungen in eine (demokratische) Gemeinschaft zu integrieren, ohne dass dies als Widerspruch erscheinen soll,

- aufgrund von Solidarisierungsdruck oder emotionaler Verbundenheit eine nahestehende Person oder Gruppe gegen Kritik in Schutz genommen werden soll,

- die Angst besteht, dass die Problematisierung von Antisemitismus bei nahestehenden Menschen zu einem Zerbrechen der Beziehung führen könnte,

22 So erscheint z.B. die These des Historikers Norbert Frei (vgl. Frei 2005: 145ff.), der bezüglich der Abwehr der »Kollektivschuldthese «im Nachkriegsdeutschland (und offenbar in Anschluss an die Freudschen Überlegungen zur »Verneinung«, vgl. Freud 1975) nahelegt, dass Schuldgefühle durchaus verbreitet waren, in seiner Pauschalität fragwürdig. Plausibler wäre es anzunehmen, dass das Vertreten der These der Kollektivschuld mit ganz unterschiedlichen Weil-Motiven verknüpft war. (vgl. z.B. Brunner 2011: 16, Reemtsma 2003). 
- die Sorge besteht, dass die Problematisierung von Gewalt und Antisemitismus Auswirkungen auf das eigene soziale Prestige bzw. den eigenen sozialen Status haben kann,

- ein strategisches Interesse daran besteht, die soziale Sanktionierung der eigenen gewaltvollen, rassistischen oder antisemitischen Handlungen oder Einstellungen zu neutralisieren und Diskursverschiebungen bezüglich der Grenzen des Sagbaren vorzunehmen.

\section{Überindividueller Charakter von Abwehrhandlungen}

Weiterhin wurde in der Auseinandersetzung mit soziologisch fundierten Abwehrkonzepten deutlich, dass Abwehrhandlungen als soziale Handlungen mit kulturtheoretischen Überlegungen in Verbindung gebracht werden können. So wurde in der Auseinandersetzung mit der Theorie der Kommunikationslatenz argumentiert, dass Abwehrhandlungen als Lösungsstrategien gesellschaftlicher Konflikte tradiert werden, wenn sie erfolgreich sind. Dies kann als gesellschaftlicher Lernprozess beschrieben werden, der zur Institutionalisierung von Abwehrrhetoriken führt. Demgegenüber betont die diskursive Psychologie, dass Individuen Abwehrargumentationen nicht individuell erlernen müssen, sondern sich in ihren Versuchen anti-antisemitischen Interventionen zu begegnen aus einem Pool an gesellschaftlich verfügbaren Skripten bedienen können, die es allesamt ermöglichen, Diskurse über Antisemitismus abzubrechen. Beide Argumentationen ergänzen sich hierbei gegenseitig.

\section{Abwehrhandlungen als funktionale Handlungen}

Zusammenfassend lässt sich sagen, dass fast alle präsentierten Ansätze gemein haben, dass sie Abwehrhandlungen als funktionale Handlungen eines besonderen Typus beschreiben. Das heißt, dass sie Abwehrhandlungen als bewusste oder unbewusste Form der Selbst- und Fremdtäuschung verstehen, die der Verdeckung eines (absehbaren oder manifesten) Konfliktes dient. Das der Handlung zugrunde liegende Motiv kann hierbei nicht offen kommuniziert werden, da genau diese Kommunikation das psychische System oder eine soziale Beziehung destabilisieren würde. Dieser verdeckt instrumentelle Charakter der Abwehr führt nach Adorno und Habermas zu einer unauffälligen Verletzung gesellschaftlich gültiger Normen, insbesondere der Norm der Wahrhaftigkeit. Adorno spricht dies an, wenn er feststellt, dass fraglos richtige Fakten für die eigene Abwehrargumentation instrumentalisiert würden. 
Es komme somit zu einem »Missbrauch der Wahrheit«. Beide Autoren stellen fest, dass Abwehrhandlungen als verdeckt strategische Handlungen deshalb erfolgreich sind, weil sie mit gesellschaftlich gültigen Normen auf beiläufige und vor allem unauffällige Weise brechen. Mit Matza und Sykes wäre diesbezüglich zu ergänzen, dass dem strategischen Bemühen von abwehrenden Akteuren auch die Deutungsbedürftigkeit sozialer Situationen und Normen entgegenkommt. D.h., sie haben Aussicht auf Erfolg, weil jede Situation, jedes Handeln immer auch anders gedeutet werden kann als von denjenigen, die (antisemitische) Gewalt problematisieren.

Für die empirische Sozialforschung ergibt sich aus diesen Befunden allerdings ein Problem. Will sie Handlungen, die auf die Dethematisierung von Antisemitismus hinauslaufen, als funktionale Abwehrhandlungen ausweisen, so muss sie plausibel machen, dass mit ihnen eine Motivlage verbunden ist, die zugleich aber im Sprechen der von den in den antisemitischen Konflikt Involvierten nicht oder nur indirekt zum Ausdruck gebracht wird. Entsprechende Analysen sind zweifelsohne oftmals nicht möglich oder riskant, wenn entsprechende Interpretationen nur eingeschränkt mit Evidenz gedeckt werden können. Dass zudem nicht jede Handlung, die Abwehrargumentationen reproduziert, als funktionale Handlung gedeutet werden muss, wurde zudem in Anschluss Kempf und Biskamp gezeigt, die argumentieren, dass Abwehrhandlungen auch zu einer Prägung von Kultur führen können. Mit der Abwehr verknüpfte Deutungsmuster (z.B. die Argumentation, dass es keinen Antisemitismus mehr gäbe) sind demnach dazu geeignet einen doxischen Charakter anzunehmen. Deshalb scheint es theoretisch notwendig, eine Unterscheidung zwischen Abwehrargumentationen als Ausdruck verdeckt strategischen Handelns und der Verwendung von Abwehrargumentationen ohne verdeckt strategischen Interessen vorzunehmen. Beide Formen lassen sich hierbei in Anschluss an Max Weber unter den Begriff sozialen Handelns fassen. Im ersten Fall sind entsprechende Argumentationen als zweckrationale Handlungen zu bezeichnen (vgl. Weber 1921: 12). Unter zweckrationalem Handeln versteht Weber ein Handeln, das unter Abwägung möglicher (argumentativer) Mittel dazu dient, bestimmte Ziele $\mathrm{zu}$ erreichen. In Anschluss an die theoretischen Befunde wäre dies noch dahingehend zu ergänzen, dass der Sinn solcher Abwehrhandlungen allerdings bewusst oder unbewusst verborgen wird. Demgegenüber definiert Weber als wertrationales Handeln ein Handeln, das auf den Glauben an den »unbedingten Eigenwert eines bestimmten Sichverhaltens « (ebd.) beruht. Die Zurückweisung von anti-antisemitischen Interventionen, bzw. die Dethematisierung von Anti- 
semitismus erfolgt hierbei auf Basis von Überzeugungen, die durch die gesellschaftliche Tradierung von Abwehrargumentationen (mit) geprägt worden sind und sich nicht auf andere Motive zurückführen lassen. Die Zurückweisung anti-antisemitischer Interventionen kann dann z.B. als Ausdruck der festen Überzeugung interpretiert werden, dass solche Interventionen falsch sind oder auf falschen Prämissen beruhen. Dies bedeutet nicht, dass diese Überzeugungen selbst unproblematisch sind und nicht kritisiert werden können. Denn der Effekt von Abwehrrhetoriken als individuell funktionale Handlungen und die auf stereotypen Wahrnehmungs- und Argumentationsmustern beruhende Zurückweisung anti-antisemitischer Interventionen, ist im Grunde derselbe. Beide führen dazu, dass antisemitische Äußerungen hingenommen, akzeptiert oder sogar befürwortet werden dienen. Diese Wirkungsweise von Abwehrhandlungen kann auf folgende Weise illustriert werden:

\section{Abbildung 2}

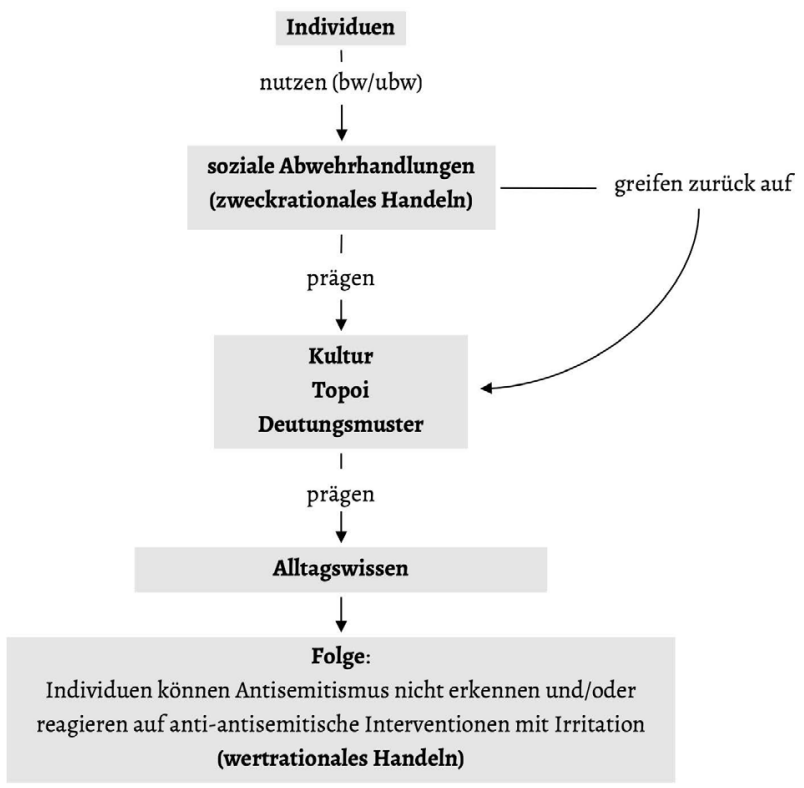


